\title{
Monitoring the hard X-ray sky with SuperAGILE
}

M. Feroci ${ }^{1}$, E. Costa ${ }^{1}$, E. Del Monte ${ }^{1}$, I. Donnarumma ${ }^{1}$, Y. Evangelista ${ }^{1}$, I. Lapshov ${ }^{1,15}$, F. Lazzarotto ${ }^{1}$, L. Pacciani ${ }^{1}$, M. Rapisarda ${ }^{12}$, P. Soffitta ${ }^{1}$, G. Di Persio ${ }^{1}$, M. Frutti ${ }^{1}$, M. Mastropietro ${ }^{1,21}$, E. Morelli ${ }^{8}$, G. Porrovecchio ${ }^{1}$, A. Rubini ${ }^{1}$,

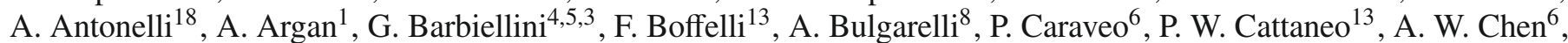
V. Cocco ${ }^{2}$, S. Colafrancesco ${ }^{14,17}$, S. Cutini1 ${ }^{14}$, F. D’Ammando ${ }^{1,2}$, G. De Paris ${ }^{1}$, G. Di Cocco ${ }^{8}$, G. Fanari ${ }^{14}$,

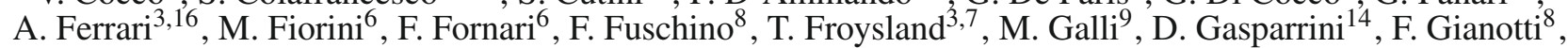

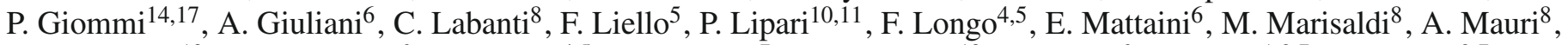
F. Mauri ${ }^{13}$, S. Mereghetti ${ }^{6}$, E. Moretti ${ }^{4,5}$, A. Morselli ${ }^{7}$, A. Pellizzoni ${ }^{19}$, F. Perotti ${ }^{6}$, G. Piano ${ }^{1,2,7}$, P. Picozza ${ }^{2,7}$, M. Pilia ${ }^{19}$, C. Pittori ${ }^{14}$, C. Pontoni ${ }^{3,5}$, B. Preger ${ }^{14}$, M. Prest ${ }^{5}$, R. Primavera ${ }^{14}$, G. Pucella ${ }^{12}$, A. Rappoldi ${ }^{13}$, E. Rossi ${ }^{8}$, S. Sabatini ${ }^{2}$, P. Santolamazza ${ }^{14}$, M. Tavani ${ }^{1,2,7,3}$, S. Stellato ${ }^{14}$, F. Tamburelli ${ }^{14}$, A. Traci ${ }^{8}$, M. Trifoglio ${ }^{8}$, A. Trois ${ }^{1}$, E. Vallazza ${ }^{5}$, S. Vercellone ${ }^{20}$, F. Verrecchia ${ }^{14}$, V. Vittorini ${ }^{1,3}$, A. Zambra ${ }^{3,6}$, D. Zanello ${ }^{10,11}$, and L. Salotti ${ }^{17}$

${ }^{1}$ INAF - IASF Roma, via del Fosso del Cavaliere 100, 00133 Roma, Italy e-mail: marco.feroci@iasf-roma.inaf.it

2 Dipartimento di Fisica, Universitá Tor Vergata, via della Ricerca Scientifica 1, 00133 Roma, Italy

3 Consorzio Interuniversitario Fisica Spaziale (CIFS), villa Gualino - v.le Settimio Severo 63, 10133 Torino, Italy

4 Dip. Fisica, Università di Trieste, via A. Valerio 2, 34127 Trieste, Italy

INFN Trieste, Padriciano 99, 34012 Trieste, Italy

INAF - IASF Milano, via E. Bassini 15, 20133 Milano, Italy

INFN Roma Tor Vergata, via della Ricerca Scientifica 1, 00133 Roma, Italy

INAF - IASF Bologna, via Gobetti 101, 40129 Bologna, Italy

ENEA Bologna, via don Fiammelli 2, 40128 Bologna, Italy

INFN Roma 1, p.le Aldo Moro 2, 00185 Roma, Italy

1 Dip. Fisica, Università La Sapienza, p.le Aldo Moro 2, 00185 Roma, Italy

ENEA Frascati, via Enrico Fermi 45, 00044 Frascati(RM), Italy

3 INFN Pavia, via Bassi 6, 27100 Pavia, Italy

ASI Science Data Center, ESRIN, 00044 Frascati(RM), Italy

5 IKI, Moscow, Russia

Dipartimento di Fisica, Universitá di Torino, Torino, Italy

7 Agenzia Spaziale Italiana, viale Liegi 26, 00198 Roma, Italy

18 Osservatorio Astronomico di Roma, via di Frascati 33, 00040 Monte Porzio Catone, Italy

19 Osservatorio Astronomico di Cagliari, loc. Poggio dei Pini, strada 54, 09012, Capoterra (CA), Italy

${ }^{20}$ INAF - IASF Palermo, via Ugo La Malfa 153, 90146 Palermo, Italy

${ }^{21}$ CNR, Istituto Metodologie Inorganiche e dei Plasmi, Area Ricerca Montelibretti, Italy

Received 23 July 2009 / Accepted 19 October 2009

\section{ABSTRACT}

Context. SuperAGILE is the hard X-ray monitor of the AGILE gamma ray mission, in orbit since 23 April 2007. It is an imaging experiment based on a set of four independent silicon strip detectors, equipped with one-dimensional coded masks, operating in the nominal energy range $18-60 \mathrm{keV}$.

Aims. The main goal of SuperAGILE is the observation of cosmic sources simultaneously with the main gamma-ray AGILE experiment, the Gamma Ray Imaging Detector (GRID). Given its $\sim$ steradian-wide field of view and its $\sim 15$ mCrab day-sensitivity, SuperAGILE is also well suited to the long-term monitoring of Galactic compact objects and the detection of bright transients. Methods. The SuperAGILE detector properties and design allow for a 6 arcmin angular resolution in each of the two independent orthogonal projections of the celestial coordinates. Photon by photon data are continuously available by means of experiment telemetry, and are used to derive images and fluxes of individual sources, with integration times depending on the source intensity and position in the field of view.

Results. We report on the main scientific results achieved by SuperAGILE over its first two years in orbit, until April 2009. The scientific observations started in mid-July 2007, with the science verification phase, continuing during the complete AGILE Cycle 1 and the first $\sim$ half of Cycle 2. Despite the largely non-uniform sky coverage, due to the pointing strategy of the AGILE mission, a few tens of Galactic sources were monitored, sometimes for unprecedently long continuous periods, leading to the detection also of several bursts and outbursts. Approximately one gamma ray burst per month was detected and localized, allowing for prompt multiwavelength observations. A few extragalactic sources in bright states were occasionally detected as well. The light curves of sources measured by SuperAGILE are made publicly available on the web in almost real-time. To enable a proper scientific use of these, we provide the reader with the relevant scientific and technical background.

Key words. instrumentation: detectors - X-rays: binaries - X-rays: general 


\section{Introduction}

The SuperAGILE experiment (Feroci et al. 2007) is the hard $\mathrm{X}$-ray imager of the Italian gamma-ray astronomy mission AGILE (Tavani et al. 2009). The primary instrument of the AGILE payload is the Gamma Ray Imaging Detector (GRID, Barbiellini et al. 2002), consisting of a Silicon Tracker (ST, Prest et al. 2003) and a small CsI(Tl) "mini"-calorimeter (MCAL, Labanti et al. 2009), surrounded by a plastic anticoincidence system (ACS, Perotti et al. 2006).

The main scientific goal of AGILE is the observation of the gamma-ray sky in the energy range from $50 \mathrm{MeV}$ to a few $\mathrm{GeV}$, over a field of view in excess of 2 steradian, ten years after the demise of the EGRET experiment onboard the Compton Gamma Ray Observatory. Several classes of celestial sources are known to emit gamma-rays in this energy band, most notably rotationpowered pulsars, active galactic nuclei (especially blazars), supernova remnants, and, occasionally, gamma-ray bursts. Other types of sources, such as galactic microquasars, low and high mass X-ray binaries or magnetars are expected to possibly emit gamma-rays in some special conditions (e.g., from shocks in the jet or in the accretion flow), but none of them had been unambiguously classified as a gamma-ray source prior to the observations by AGILE or Fermi-GLAST (with the exception of the debated cases of LS 5039 and LSI +61 303).

The SuperAGILE experiment was designed to guarantee the hard X-ray (18-60 keV) monitoring in the tens of mCrab sensitivity range of the central $\sim$ steradian of the GRID field of view, aiming to identify the hard X-ray sources and/or conditions of special interest to the GRID, providing accurate ( $\sim \operatorname{arcmin})$ localizations and allowing for multi-band observations. In addition to that, SuperAGILE independently acts as a wide field monitor for bright Galactic sources, with photon by photon transmission and $5-\mu$ s time accuracy.

A detailed description of the SuperAGILE experiment (preflight) may be found in Feroci et al. (2007), while information about the early inflight operation and technical performance may be found in Feroci et al. (2008a). Here we summarize the main instrument properties relevant to the aim of the present paper, which is providing an overview of the main scientific results achieved by the experiment during the first 20 months of observations from space, as well as guidelines and limits for the proper scientific use of the SuperAGILE data.

SuperAGILE is a coded-mask experiment devoted to imaging the transient hard X-ray sky by means of a set of four onedimensional Silicon microstrip detectors, encoding the sky in two orthogonal directions. The field of view (FOV) of each pair of detectors is rectangular, $107^{\circ} \times 68^{\circ}$ at zero response (Fig. 1). Thus, the central $68^{\circ} \times 68^{\circ}$ region is encoded in both directions $(2 \times 1 \mathrm{D})$, while the peripheral $\sim \pm 20^{\circ}$ of the FOV are coded in one dimension only. Because of severe limitations in the design and operation level (see Feroci et al. 2007, 2008a), SuperAGILE operates in the non-optimal (for a Silicon detector) energy range from 18 to $60 \mathrm{keV}$, being noise-limited at the low energy bound and efficiency limited at the upper bound, and behind a $5 \mathrm{~mm}$ thick shield of plastic scintillator (part of the AGILE ACS). The energy resolution is $\sim 8 \mathrm{keV}$ full width at half maximum, energy-independent. The one-dimensional point spread function (PSF) is 6 arcmin on-axis and the point source location accuracy reaches $1-2$ arcmin for detections above $\sim 10 \sigma$, including a $\sim 1$ arcmin systematic contribution from the AGILE satellite attitude reconstruction. The SuperAGILE on axis 5- $\sigma$ sensitivity is $\sim 20 \mathrm{mCrab}$ for a $50 \mathrm{ks}$ net exposure (independently in each direction, i.e., half of the area of the experiment). Because of the

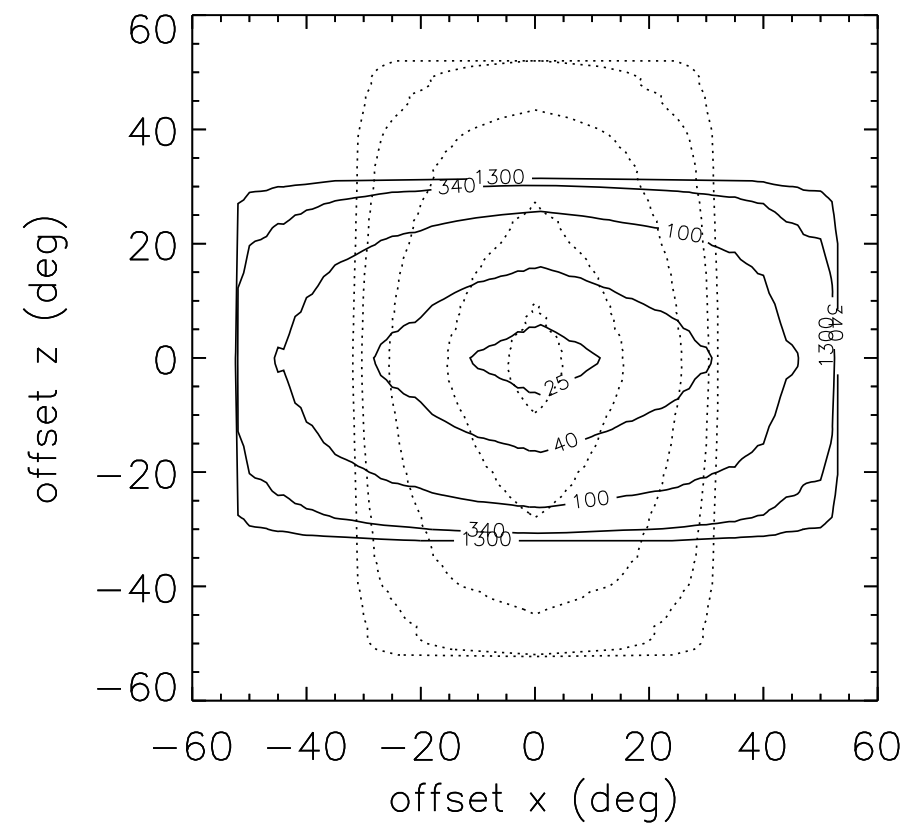

Fig. 1. Representation of the field of view of the two pairs of SuperAGILE detectors, encoding two directions in the sky. Here we show with solid lines the sensitivity contours of one pair of detectors (i.e., one coding direction, either $X$ or $Z$ ). The contours in dotted lines represent the sensitivity of the other pairs of detectors, rotated by $90^{\circ}$, providing the other coordinate. Sensitivity is given in mCrab $(20-60 \mathrm{keV})$ at $5-\sigma$ confidence level, over a $50 \mathrm{ks}$ integration time, for one direction only (half of the experiment area).

triangular response of the collimator, the FOV at half sensitivity with $2 \times 1 \mathrm{D}$ imaging is approximately $30^{\circ} \times 30^{\circ}$. In each direction, the sensitivity is superior to $100 \mathrm{mCrab}(5 \sigma, 50 \mathrm{ks}$, two detectors only) within the central $40^{\circ} \times 80^{\circ}$ region (see Fig. 1). The on-axis effective area is $250 \mathrm{~cm}^{2}$ (total of 4 detectors, at $20 \mathrm{keV}$ ). The experiment background is dominated by the diffuse X-rays. Particle-induced background is effectively rejected by the amplitude discrimination and the anticoincidence logic of the ACS, which produces a large dead-time effect when crossing the south Atlantic anomaly, preventing in practice scientific observations in this orbital phase (see, e.g., Feroci et al. 2008a).

The SuperAGILE experiment entered its nominal observing phase in mid-July 2007, after the successful completion of its commissioning phase. Owing to the inaccessibility of the Crab Nebula in June, caused by the Sun constraints of the spacecraft, the first light of the experiment was performed on the Vela region, leading to the detection of the Galactic binary system GX 301-2. Between July and October 2007, the experiment was calibrated in flight, using the Crab Nebula to scan several positions in the FOV, as a part of its science verification phase. The latter was completed by the end of November 2007 with the observation of the Vela field, the Galactic center and the Cygnus region. On December 2007, the AGILE observing cycle 1 started, which typically consisted of continuous exposures of 4 weeks of the same field. In contrast to those of GRID, the SuperAGILE data of Cycle 1 are not open to guest observers. However, light curves of the sources detected by SuperAGILE are made available for public distribution in almost real-time by means of a web page (see Sect. 6).

In the following sections we describe the scientific data products of SuperAGILE (Sect. 2), review the status of experiment calibration and pointing strategy (Sects. 3 and 4), and overview the main scientific observations carried out over the 
first $\sim 20$ months of nominal operations in orbit (Sect. 5), including a preliminary list of the detected sources.

\section{The SuperAGILE data analysis and scientific products}

The SuperAGILE experiment (hereafter SA) transmits photonby-photon data to the AGILE telemetry and then to the ground. For each detected event, information is available about its time of arrival (with $2 \mu$ s resolution and $5 \mu$ s absolute accuracy), energy ( 64 channels, formally between 0 and $\sim 90 \mathrm{keV}$ ), and position on the detectors (strip address between 1 and 6144, including the detector information).

The SA telemetry is downloaded from the spacecraft to the ground station in Malindi (Kenya) once per orbit (which is approximately $100 \mathrm{~min}$ long). It is then transmitted to the Telespazio Fucino Space Center and to the ASI Science Data Center in Frascati, where it is both locally processed and transmitted to the SuperAGILE Team in Rome (see Trifoglio et al. 2008, for a detailed description of the AGILE data flow and processing). An automatic data processing pipeline, SASOA (Lazzarotto et al. 2008, 2009), extracts high-level products and scientific information from the SA data. One-dimensional images of the sky in each of the two SA coordinates are created using data of the entire orbit. This is intended to provide an unbiased "picture" of the hard X-ray sky in the experiment field of view, during that orbit. The results of this analysis are automatically stored in a database, from where orbital light curves of the sources positively detected can be retrieved over the entire mission lifetime. The same procedure runs automatically on a daily timescale, following the same approach.

A refined analysis can be performed by the user on sources of interest. It consists of performing the same above mentioned procedures, but applying specific time and/or coordinates and/or energy selections. For individual sources, optimized results are obtained, for example, by selecting the SA data for time periods when the specific source of interest was not occulted by the Earth. To these, additional time and energy selections can be applied, resulting in energy-selected light curves of the source. Energy spectra can also be obtained by performing the imaging procedure iteratively on pre-determined energy bins, and extracting for each of them the source intensity from the combined images in the $X$ and $Z$ detectors. The results are then stored in a standard fits pha file, to be used in the XSPEC spectral analysis package, together with the proper instrument response matrix. The latter is accumulated for each pair of detectors ( $X$ and $Z$ ). The response matrix is dependent on the source position in the FOV and is then computed for each spectrum.

A crucial step in the SA scientific analysis is the attitude correction. The one-dimensional SuperAGILE images are severely affected by the satellite attitude variations, which are as large as $0.1 \%$, meaning that the satellite pointing direction varies by an amount equal to the SA angular resolution every second. This is not similarly critical for the GRID, which has full 2D imaging capability and an angular resolution in the range of $\sim 4^{\circ}$. The detected position of every SA event needs to be corrected to its expected location using the attitude information from the AGILE star sensors, available in telemetry with a $10 \mathrm{~Hz}$ frequency and a 1 arcmin accuracy, on each of the three satellite axes.

In a coded-mask experiment, the attitude correction of an individual photon must make an assumption about its incoming direction. Unlike one would intuitively expect for a onedimensional imager such as SA, the one-dimensional shift

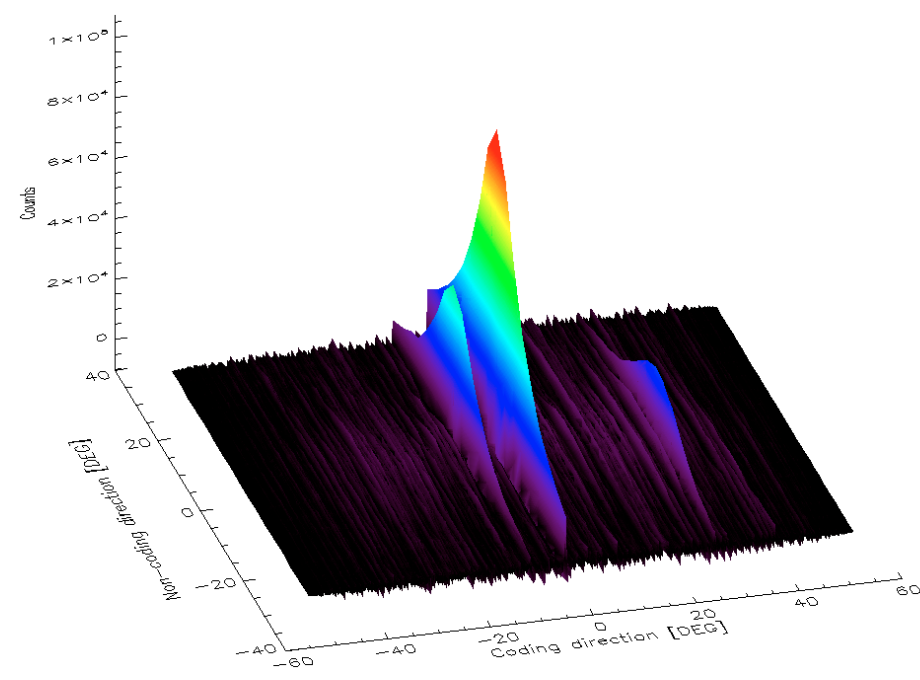

Fig. 2. A bi-dimensional image obtained by the SuperAGILE Z detectors observing the Vela field on DOY 14, 2009. The coding direction shows the typical arcmin-resolution, while the non-coding direction displays the counts obtained after different attitude corrections (see text for details). The peak positions in the Non-coding direction identify the source position along that coordinate. The three peaks correspond to the sources 1A 1118-61 (brightest), GX 301-2 (second brightest, on the left), and Vela X-1 (dimmest, on the right).

compensating an attitude variation depends not only on the projection of the source position along the coding direction, but also along its non-coding direction. In practice, the attitude variation provides an additional encoding to the photons, also in the direction where the detector has no imaging capabilities. This implies that the a priori assumptions about the incoming direction of the photon (and the attitude correction thereof) and the search for point sources in the FOV must be completed in a twodimensional space, instead of the more "natural" one-dimension. One of the two directions ("coding") has a resolution of 3 arcmin, as determined by the size of the sky pixel of the experiment. The second one ("non-coding") has a resolution (hereafter "zone") determined by a trade-off between the sensitivity of the SA point spread function to the attitude variation and the processing time. In the current version of the SA scientific software, the angular size of a non-coding zone is set to be $4^{\circ}\left( \pm 2^{\circ}\right)$. A positive consequence is that each SA detector becomes a twodimensional imager, but at the expenses of the signal-to-noise ratio. We show this effect in Fig. 2, where a mask-deconvolved image ("sky image") is presented for a field including three sources. In the coding direction, the source position shows up as a sharp peak (PSF-wide), while in the non-coding direction the true position corresponds to a broad but still identifiable peak (as a function of the "zone", in the attitude correction algorithm). In the SA data processing, a catalogued source identification is then completed by requesting that the SA measured position lies within \pm 3 arcmin in the coding direction, and within \pm 1 zone (i.e., $\pm 4^{\circ}$ ) in the non-coding direction.

The software package implementing this imaging procedure, called EMI (Enhanced Multi sky Imaging), is applied to the one-dimensional sky images obtained by coadding data from homologous detectors (i.e., the two pairs of detectors encoding the $X$ and $Z$ directions) to detect, localize, and identify the significant sources in the field, independently in both coordinates. The source flux is derived by integrating the peak counts over a PSF width and then normalizing them to the net source exposure (that is, excluding the times when the source was occulted by 
the Earth or the satellite was passing through the South Atlantic Anomaly) and to the effective area at the relevant FOV position. The resulting normalized intensity, in units of counts $\mathrm{cm}^{-2} \mathrm{~s}^{-1}$, is a measurement of the source flux and is then independent of the source position in the FOV.

The EMI procedure is able to detect and iteratively subtract the source peaks, to identify weaker sources, but in doing so it does not simulate the instrumental response to the source and is then not suited to the subtraction of the coding noise because of the partial coding of the detected sources (the entire SuperAGILE field of view is indeed only partially coded, except for the on-axis direction). For this purpose, a dedicated IROSlike (Iterative Removal Of Sources) procedure was developed, which fits the complete imaging response to a detected source, to iteratively subtract it from the image and search for the subsequent, dimmer source. Compared to similar packages developed for past experiments, the IROS procedure for SA must be able to account for its several departures from ideality, making its operation far more complex than usual. The attitude variations in the AGILE spacecraft amount to one SuperAGILE PSF every second $\left(0.1^{\circ} \mathrm{s}\right)$, within a range of $\sim 1^{\circ}(\sim 40$ times the $\sim 1.5 \operatorname{arcmin}$ SA point source location accuracy, see next section). The electronic noise in the front-end electronics is large because of the high operative temperature (always in the range $\sim 25-30^{\circ} \mathrm{C}$ ) and is variable because of the large temperature variations $\left(\sim 3-5^{\circ} \mathrm{C}\right)$ along the orbit. The electronic noise critically affects the imaging capabilities for signal amplitudes close to the low energy threshold (see Pacciani et al. 2008a, for an extensive discussion). All of these effects, combined also with the one-dimensional imaging capability of the experiment, cause significant instabilities in the simulation of the observed sky by the IROS procedure. Owing to these intrinsic difficulties, all of the above experiment characteristics had to be studied and understood on the basis of the flight data, and modelled by the software. This data analysis package, named EMIROS, is now close to completion and will be used to improve the current imaging sensitivity of the experiment.

The analysis presented in this paper is based on the EMI data processing only, implying that it has a limiting sensitivity that is poorer than the intrinsic experimental potential. The coding noise of strong sources in the image in some cases affects the flux estimate of the weaker sources, and this is taken into account by adding a systematic uncertainty. This issue is discussed in the next section.

\section{Status of experiment calibration}

The SuperAGILE experiment was calibrated on the ground at different stages of its integration, from the "naked" silicon detectors to the full experiment inside the spacecraft in-flight configuration. Because of its very high angular resolution (6 arcmin) over a large field of view ( $\sim 1$ steradian), but poor energy resolution $(\sim 8 \mathrm{keV}$, full width at half maximum) over a narrow bandpass (18-60 keV), SuperAGILE acts mostly as an X-ray imager. For this reason, most of the calibration and software efforts were devoted to both its imaging properties and the calibration of the integrated effective area.

During the ground calibrations, the wide field of view of the experiment was scanned using radioactive sources of different energies . Similarly, in-orbit a series of 251 -day pointings at the beginning of the mission were devoted to observing the Crab Nebula at different positions of the FOV. These data were used to verify and refine the results of the ground calibrations, in terms of point source location accuracy and effective area, as a function of the source position in the FOV. An onboard electronic circuit allows us to monitor and verify periodically the stability of the gain and linearity of the experiment. A detailed description of the ground and in-flight calibrations, their analysis, results, and the estimation of systematic effects may be found in Donnarumma et al. (2006), Evangelista et al. (2006), and Feroci et al. (2008a). Here we summarize the results most relevant to the data interpretation.

Because of the above-mentioned time/temperaturedependent threshold effects combined with the poor energy resolution (see Pacciani et al. 2008a, for details), the energy range between 17 and $20 \mathrm{keV}$ is very difficult to calibrate accurately, yet it is an important one, due to the effects of the silicon quantum efficiency and the energy spectra of cosmic sources, which are both enhanced by the low energy response. This energy range is then used only when the short-term signal-to-noise ratio is high enough to permit us to neglect the threshold effects, on timescales shorter than the typical temperature variations. This is usually achieved on short timescale transients, such as gamma-ray bursts or type I X-ray bursts, while for longer integrations of the persistent emission of steady sources we chose to limit our analysis to energies above $20 \mathrm{keV}$.

The point source location accuracy (PSLA) was verified inflight by detecting tens of known sources (see Sects. 5.1 and 5.5). By using hundreds of detections in different positions of the field of view, the PSLA was determined to be $1.5 \mathrm{arcmin}$ ( $90 \%$ containment radius) for detections of a statistical significance higher than $10 \sigma$. The imperfect knowledge of the true satellite attitude from the star sensors data introduces a large systematic contribution ( $\sim 1$ arcmin). In principle, one could also expect a contribution to the PSLA by the angular extension of the Crab Nebula, found to be $\sim 1-2$ arcmin below $10 \mathrm{keV}$. The contribution of this extension can be quantitatively neglected when the 6-arcmin point spread function of SA is considered. We also verified that the determination of the PSLA provides consistent results both using the localizations of the Crab Nebula only, and the localization of the other known sources (see Sect. 5.1), thus suggesting a low surface brightness of the outer regions of the Crab Nebula with the SA bandpass.

The source flux reconstruction is still significantly affected by the systematics induced by a non perfect calibration of the threshold and thermal-noise effects of the individual channels, as well as by coding noise effects when a strong source (in terms of detected counts) is in the field. Based on the mask design, the amplitude of the coding noise induced by a source in other positions of the FOV is expected to be at most $8 \%$ of its peak flux.

Using the raster scan of the experiment field of view with the Crab Nebula, we were also able to study the systematics of the source flux determination at the different positions of the FOV, for the cases of only one or a dominant source in the field. We selected positions in the SA field of view where the exposed area was at least $20 \%$ of the on-axis value in at least one of the two directions $(X$ or $Z$ ). By means of the automatic orbital analysis, we then used the Crab observations to estimate the systematic uncertainty in the source flux determination. A set of $\sim 500$ orbital detections of the Crab Nebula were found to satisfy the above criterion, and provide a (statistically) constant Crab value in the 20-60 keV energy range of 0.1511 counts $\mathrm{cm}^{-2} \mathrm{~s}^{-1}$, provided that a systematic uncertainty equal to $8 \%$ of the source counts is added in quadrature to the statistical uncertainty in the individual measurement. In Fig. 3, we show, as examples, three $\sim 1$-week stretches of orbital Crab detections, in three different positions in the field of view, demonstrating the source flux stability to within the quoted uncertainties. 


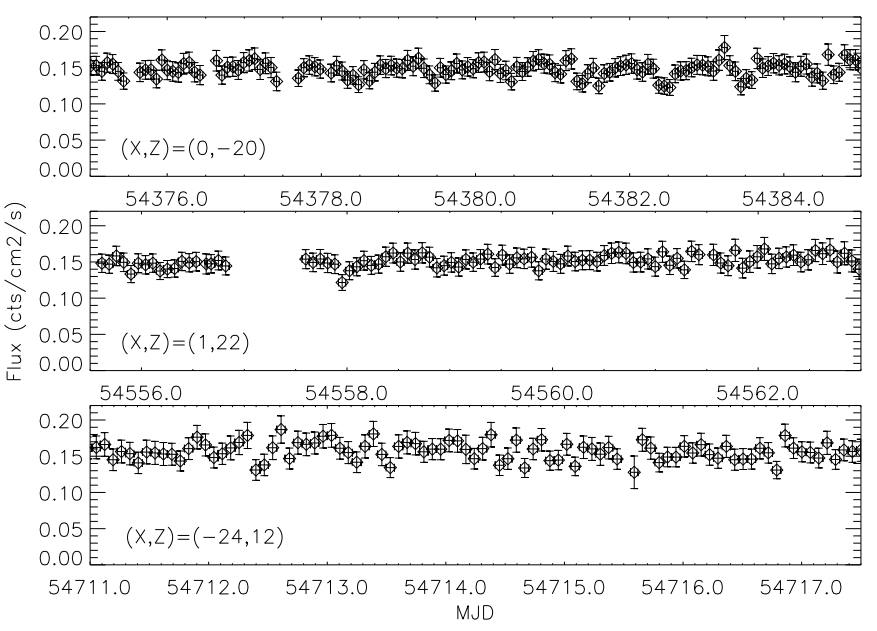

Fig. 3. Examples of $\sim$ week-long sequences of automatic orbital detection of the Crab for different positions in the SA field of view.

The calibration of the orbital determination of the source flux was also successfully tested by comparing the simultaneous observations of variable sources with SA and Swift/BAT, using the publicly available BAT light curves ${ }^{1}$ in nearly the same energy range (BAT reports the $15-50 \mathrm{keV}$ energy range). The calibration of this type of data is particularly relevant to the use of the SA orbital light curves of sources in public distribution (see Sect. 6).

\section{The AGILE pointing strategy and the SuperAGILE sky coverage}

The AGILE satellite has fixed solar panels and the spacecraft attitude control system requires that the solar panels are oriented in the Sun direction to within $\pm 1^{\circ}$ at any time. Therefore, the range of pointing directions that satisfies this Sun constraint is included in a $2^{\circ}$ wide circle, centered at $90^{\circ}$ from the Sun, and it drifts by $1^{\circ}$ per day to ensure that the constraint is always satisfied. This odd condition is less critical for the AGILE scientific payload than it would be in other cases, because of the very large field of view of the GRID and SA experiments.

From the scientific point of view, the primary experiment onboard the AGILE mission is GRID, and the optimization of its observing program is the motivation behind the pointing strategy of the AGILE mission (e.g., Pittori et al. 2009). The effective field of view of GRID is significantly wider (by almost a factor of 2) than that of SuperAGILE, implying that looser pointing constraints are required to reach a reasonably uniform coverage of the sky. As a result, the integrated SuperAGILE exposure map acquired after the period July 2007 - April 2009 is largely nonuniform and mostly tracks the average AGILE boresight position history. In Table 1, we list the sky regions at which the AGILE mission was pointed during the first $\sim 2$ years. Updated AGILE pointing information for both past and future observations may be obtained at the web site of the AGILE Data Center ${ }^{2}$, part of the ASI Science Data Center (ASDC).

Taking into account the SA effective area as a function of the off-axis angle, we can then derive the exposure map, which represents the cumulative distribution of the product of the net exposure time and the effective area, in units of $\mathrm{cm}^{2} \mathrm{~s}$, as shown in

\footnotetext{
${ }^{1}$ http://swift.gsfc.nasa.gov/docs/swift/results/ transients/

${ }^{2}$ http://agile.asdc.asi.it/
}

Table 1. A list of the main AGILE pointings during the first two years.

\begin{tabular}{|c|c|c|c|c|}
\hline Sky Region & Date start & Date stop & RA & Dec \\
\hline \multirow[t]{6}{*}{ Vela field } & $2007-07-13$ & $2007-07-24$ & 158.0 & -60.2 \\
\hline & 2007-07-30 & 2007-08-01 & 150.8 & -70.2 \\
\hline & 2007-08-02 & 2007-08-12 & 176.0 & -66.1 \\
\hline & 2007-08-13 & 2007-08-22 & 195.6 & -66.6 \\
\hline & $2007-08-23$ & $2007-08-27$ & 217.0 & -64.4 \\
\hline & 2008-01-08 & 2008-02-01 & 147.0 & -62.5 \\
\hline \multirow[t]{10}{*}{ Galactic anti-center } & 2007-08-01 & 2007-08-02 & 37.1 & 12.7 \\
\hline & $2007-08-12$ & $2007-08-13$ & 47.4 & 16.1 \\
\hline & $2007-08-22$ & $2007-08-23$ & 57.1 & 18.6 \\
\hline & 2007-09-01 & 2007-09-04 & 68.1 & $20.6^{(*)}$ \\
\hline & 2007-09-12 & 2007-09-13 & 78.5 & 21.7 \\
\hline & 2007-09-15 & $2007-10-13$ & 92.3 & $21.9^{(*)}$ \\
\hline & $2007-10-22$ & $2007-10-23$ & 120.5 & 18.9 \\
\hline & 2007-11-01 & $2007-11-02$ & 130.6 & 16.3 \\
\hline & 2008-03-30 & 2007-04-10 & 107.8 & $26.7^{(*)}$ \\
\hline & 2009-03-31 & 2009-04-07 & 102.7 & 31.7 \\
\hline \multirow[t]{7}{*}{ Galactic Center } & 2007-08-27 & 2007-08-27 & 236.6 & -41.9 \\
\hline & $2007-10-13$ & $2007-10-22$ & 290.9 & -18.9 \\
\hline & $2007-10-23$ & $2007-10-24$ & 301.2 & -17.1 \\
\hline & 2008-03-01 & 2008-03-30 & 254.7 & -39.8 \\
\hline & 2008-09-10 & $2008-10-10$ & 256.5 & -28.5 \\
\hline & 2009-02-28 & $2009-03-25$ & 247.0 & -29.0 \\
\hline & 2009-03-25 & 2009-03-31 & 275.7 & -30.5 \\
\hline \multirow[t]{7}{*}{ Cygnus field } & 2007-11-02 & $2007-12-01$ & 296.9 & 34.5 \\
\hline & 2007-12-01 & $2007-12-16$ & 315.0 & 45.0 \\
\hline & $2008-05-10$ & 2008-06-09 & 304.3 & 36.0 \\
\hline & 2008-06-15 & $2008-06-30$ & 323.2 & 50.1 \\
\hline & 2008-10-31 & $2008-11-30$ & 295.5 & 35.6 \\
\hline & $2008-11-30$ & 2009-01-12 & 327.7 & 49.0 \\
\hline & 2009 & 2009-02-28 & 325.7 & 68.1 \\
\hline \multirow[t]{2}{*}{ Musca field } & 2008-02-14 & 2008-03-01 & 191.9 & -71.9 \\
\hline & 2008-08-15 & 2008-08-31 & 175.3 & -74.1 \\
\hline \multirow[t]{2}{*}{ Aquila field } & $2008-10-17$ & $2008-10-31$ & 291.0 & 10.1 \\
\hline & 2009-04-07 & 2009-04-30 & 289.9 & -1.6 \\
\hline Antlia field & 2008-06-30 & $2008-07-25$ & 161.8 & -47.7 \\
\hline Vulpecula field & 2008-04-10 & 2008-04-30 & 286.3 & 20.8 \\
\hline Virgo region & $2007-12-16$ & $2008-01-08$ & 173.4 & -0.4 \\
\hline \multirow[t]{2}{*}{ "Field 8" } & 2007-09-04 & 2007-09-12 & 51.4 & 71.0 \\
\hline & 2007-09-13 & 2007-09-15 & 74.9 & 58.3 \\
\hline North Galactic pole & 2008-04-30 & $2008-05-10$ & 250.1 & 72.5 \\
\hline \multirow[t]{2}{*}{ South Galactic pole } & 2008-02-01 & 2008-02-09 & 58.3 & -37.8 \\
\hline & 2008-02-12 & 2008-02-14 & 65.7 & -35.7 \\
\hline ToO W Comae & 2008-06-09 & $2008-06-15$ & 182.3 & 29.6 \\
\hline ToO S50716+714 & $2007-10-24$ & 2007-11-01 & 155.5 & $67.4(*)$ \\
\hline ToO Mkn 421 & 2008-02-09 & $2008-02-12$ & 251.0 & 50.3 \\
\hline \multirow[t]{2}{*}{ ToO 3C454.3 } & 2007-07-24 & 2007-07-30 & 17.8 & 36.7 \\
\hline & $2008-07-25$ & $2008-08-15$ & 22.3 & 39.1 \\
\hline ToO SGR0501+4516 & 2008-08-31 & 2008-09-10 & 61.9 & 44.1 \\
\hline ToO PKS0537-441 & 2008-10-10 & $2008-10-17$ & 98.8 & -46.8 \\
\hline ToO Carina field & 2009-01-12 & 2009-01-19 & 161.7 & -59.9 \\
\hline
\end{tabular}

Notes. ${ }^{(*)}$ Average coordinates.

Fig. 4 (top panel). The false-color exposure map in Galactic coordinates clearly shows the effect of the AGILE long exposures on the Vela and Cygnus regions, which are favored not only by the presence of several scientific and calibration targets for the GRID experiment, but also by the Sun constraints. Relatively deep exposures were also obtained of the Galactic center and anti-center regions (the latter because of the presence of the Crab Nebula), and the Virgo region, where a coordinated multifrequency campaign was performed (see Pacciani et al. 2009a). 

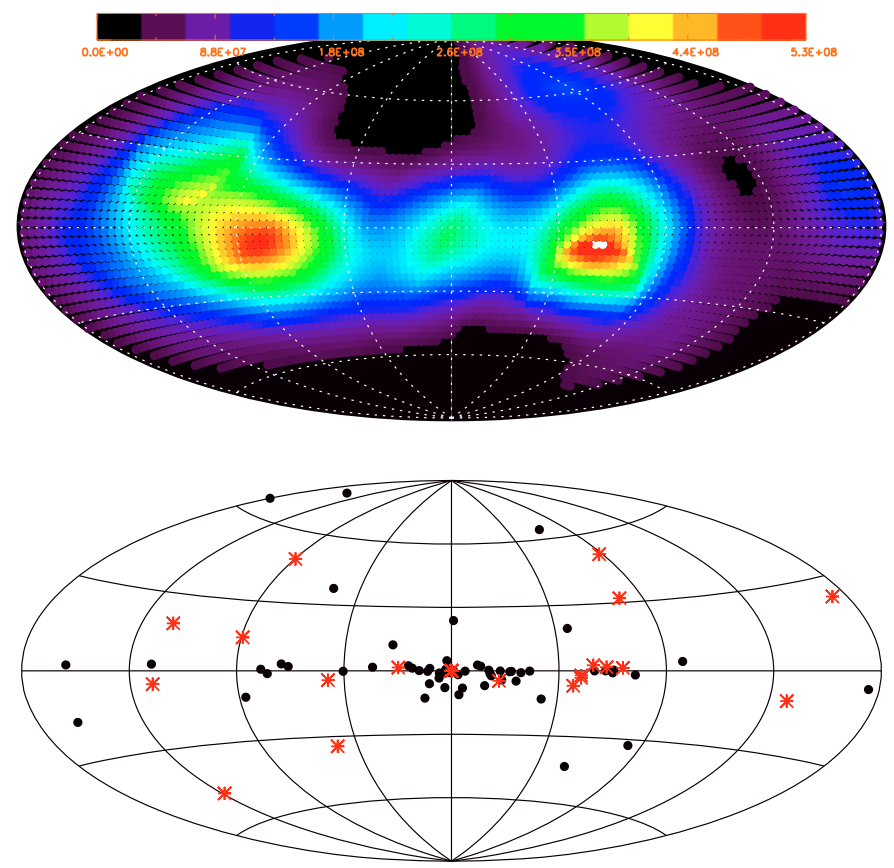

Fig. 4. Top panel: integrated exposure map, in units of the product of effective area and exposure time, to any region of the sky, over the time period July 2007 - April 2009. Bottom panel: distribution of the sources detected by SuperAGILE in Galactic coordinates. Red asterisks show the sky distribution of the localized gamma-ray bursts.

\section{Science with SuperAGILE}

The characteristics of the SuperAGILE experiment make it suited to the detection of bright transients and the extended monitoring of bright Galactic sources. The one-dimensional imaging, and the spacecraft attitude control system and its constraints ensure that it is not well suited to very long integrations (i.e., longer than $\sim 1$ week). However, because of its large field of view, SuperAGILE is able to provide month-long continuous light curves of several sources simultaneously, using bin sizes from minutes to $\sim$ days, depending on the source intensity and position in the field of view. For bright sources, timing and spectral analyses can also be carried out using the photon-by-photon data. In addition to the persistent sources, SA typically detects and localizes one gamma-ray burst per month, as well as other Galactic or extragalactic transients.

In the following sections, we report on the sources for which flux measurements with SA are available so far and provide details about noticeable regions of the sky, which represent useful case studies to illustrate the results of both automated and manual analyses of the SA data. Deeper analyses and interpretation of a few specific sources is the subject of individual papers, e.g., the case of Mkn 421 (Donnarumma et al. 2009a) or 3C 273 (Pacciani et al. 2009a), as well as the gamma-ray bursts (Del Monte et al. 2008a).

\subsection{Detected sources}

SuperAGILE observed the sky with widely varying exposure times in different regions, during its first $\sim 20$ months of observations, which were completed between mid-July 2007 and the end-April 2009, with the satellite $\sim 94 \%$ of the time in nominal pointing and the experiment in nominal configuration. The typical pointing strategy of the AGILE mission is to have month-long observations of the same field, the Sun constraints imposing a drift of the observed field by approximately $1^{\circ}$ per day. During this period, SuperAGILE was able to monitor a few tens of sources, which are typically brighter than $\sim 30$ $40 \mathrm{mCrab}$.

Table 2 summarizes the current status of the SA source detections. The results reported in the table were derived mostly from the automated daily integrations (i.e., $\sim 40 \mathrm{ks}$ net exposure each). In some other cases, specified in the "timescale" column, the reported source was detected by means of its transient emission, measured using a dedicated analysis (e.g., for Galactic transients). We specify for each source whether it was also detected by the automated analysis on an orbital timescale, implying that flux measurements for it are then publicly available at the ASDC website. We note that Table 2 is not intended to be a catalog. The analysis that produced these results was not carried out homogeneously and systematically over the full data archive and was not optimized for the source detection (see Sect. 2). In addition, shorter and longer integration times were not systematically explored. A complete reanalysis of the full SA data archive is planned with the final EMIROS software package. A complete SA source catalog will be the subject of a future publication, where a more homogeneous and complete analysis of the SA data archive will be reported.

For each source, the columns in the table report, following the name: the celestial coordinates, the average flux measured by SA in the 20-60 keV, the total exposure time, the maximum significance of the individual SA measurements (on typically daily timescales), the source type, the availability of orbital measurement data, and any notes for a given source. We note that the average flux column in the table provides the source flux measurements obtained by positive detections only (i.e., above $4 \sigma$ within a region of the SA field of view exposing no less than $20 \%$ of the on-axis effective area, even if in only one of the two SA coordinates). These numbers are biased toward high fluxes. Therefore, these flux measurements are not to be taken as an average flux level of individual sources over the 2007-2009 time interval. Similarly, we note that the results of the automated orbital analysis (see Sect. 6), also for positive detections only, are biased to even higher values, due to the lower sensitivity of the experiment on this shorter timescale. Thus, the average flux measurement of the orbital analysis is expected to be systematically higher than for the daily integrations (except for bright sources), because the analysis preferentially selects the times when the source shows a flux higher than the SA orbital sensitivity. The exposure time column is meant to provide the reader with general information about how long a specific source has been at a flux level detectable by SuperAGILE during its visibility periods. It is computed by adding the integrations during which the source was positively detected, filtering out the time when the source was occulted by Earth and the satellite passed through the South Atlantic Anomaly.

In Fig. 4 (bottom panel) we show the sky distribution, in Galactic coordinates, of the sources detected by SA and listed in Table 2. As expected, the sources are mostly located at the Galactic center, and where AGILE spent the largest fraction of its observing time, i.e., the Vela and Cygnus regions.

\subsection{The Vela region}

In this and the next section, we show the analysis of two significant fields observed by SuperAGILE, representing conditions with a few and many sources, the Vela region and the Galactic center. We use them as case studies to describe and explain the 
M. Feroci et al.: Hard X-ray sky with SuperAGILE

Table 2. The list of X-ray sources detected by SuperAGILE in the period July 2007 - April 2009.

\begin{tabular}{|c|c|c|c|c|c|c|c|c|}
\hline Name & $\begin{array}{l}\text { RA } \\
(\mathrm{deg})\end{array}$ & $\begin{array}{c}\text { Dec } \\
(\mathrm{deg})\end{array}$ & $\begin{array}{l}\text { Average detected flux } \\
\text { (mCrab) }\end{array}$ & $\begin{array}{l}\text { Total exposure } \\
(\mathrm{ks})\end{array}$ & $\begin{array}{l}\text { Max. sign. } \\
\qquad(\sigma)\end{array}$ & Type & $\begin{array}{c}\text { Orbital } \\
\text { data }\end{array}$ & Note \\
\hline SMC X-1 & 19.271 & -73.443 & 87 & 140 & 4.4 & HMXB & $\mathrm{N}$ & \\
\hline $1 \mathrm{~A} 0114+650$ & 19.511 & 65.292 & 13 & 380 & 5.9 & HMXB & $\mathrm{N}$ & 5-h flare \\
\hline X Per & 58.846 & 31.045 & 55 & 45 & 4.5 & HMXB & $\mathrm{Y}$ & \\
\hline SGR $0501+4516$ & 75.278 & 45.276 & - & - & - & SGR & $\mathrm{N}$ & Short bursts \\
\hline LMC X-4 & 83.207 & -66.370 & 50 & 400 & 10.1 & HMXB & $\mathrm{N}$ & \\
\hline Crab & 83.628 & 22.020 & 1000 & 2477 & 76.4 & PWN & $\mathrm{Y}$ & \\
\hline Vela X-1 & 135.512 & -40.557 & 603 & 1523 & 47.6 & HMXB & Y & \\
\hline GRO J1008-57 & 152.441 & -58.291 & 56 & 220 & 6.0 & HMXB & $\mathrm{N}$ & \\
\hline Mkn 421 & 166.114 & 38.209 & 40 & 140 & 9.4 & BL & $\mathrm{N}$ & \\
\hline 1A $1118-61$ & 170.238 & -61.917 & 475 & 447 & 47.6 & HMXB & Y & \\
\hline Cen X-3 & 170.300 & -60.638 & 173 & 1859 & 21.3 & HMXB & $\mathrm{Y}$ & \\
\hline 1E 1145.1-6141 & 176.869 & -61.953 & 44 & 1010 & 9.4 & HMXB & $\mathrm{Y}$ & \\
\hline NGC 4151 & 182.636 & 39.405 & 17 & 115 & 6.9 & Sy & $\mathrm{N}$ & \\
\hline GX 301-2 & 186.657 & -62.770 & 390 & 3315 & 129.9 & HMXB & $\mathrm{Y}$ & \\
\hline $3 \mathrm{C} 273$ & 187.278 & 2.052 & 24 & 740 & 21.3 & QSO & $\mathrm{N}$ & \\
\hline Cen A & 201.365 & -43.019 & 60 & 200 & 6.6 & RG & $\mathrm{Y}$ & \\
\hline 1E1547.0-5408 & 237.725 & -54.307 & - & - & - & AXP & $\mathrm{N}$ & Short bursts \\
\hline 4U 1608-522 & 243.179 & -52.423 & 126 & 130 & 8.22 & LMXB & $\mathrm{Y}$ & \\
\hline Sco X-1 & 244.979 & -15.640 & 1666 & 3594 & 103.0 & LMXB & $\mathrm{Y}$ & \\
\hline $4 U 1624-490$ & 247.011 & -49.198 & 139 & 8 & 4.6 & LMXB & $\mathrm{N}$ & \\
\hline IGR J16318-4848 & 247.966 & -48.808 & 165 & 360 & 22.6 & HMXB & $\mathrm{Y}$ & \\
\hline 4U 1626-67 & 248.070 & -67.461 & 53 & 2600 & 11.7 & LMXB & $\mathrm{Y}$ & \\
\hline 4U 1636-536 & 250.231 & -53.751 & 68 & 380 & 8.5 & LMXB & $\mathrm{Y}$ & \\
\hline GX $340+0$ & 251.448 & -45.611 & 85 & 908 & 18.5 & LMXB & $\mathrm{Y}$ & \\
\hline Her X-1 & 254.458 & 35.342 & 200 & 122 & 5.3 & HMXB & $\mathrm{N}$ & \\
\hline OAO $1657-415$ & 255.199 & -41.673 & 96 & 530 & 27.1 & HMXB & $\mathrm{Y}$ & \\
\hline GX 339-4 & 255.706 & -48.789 & 105 & 868 & 11.3 & LMXB & $\mathrm{Y}$ & \\
\hline 4U 1700-377 & 255.986 & -37.844 & 357 & 1179 & 35.8 & HMXB & $\mathrm{Y}$ & \\
\hline GX 349+2 & 256.435 & -36.423 & 117 & 3110 & 21.4 & LMXB & Y & \\
\hline 4U 1702-429 & 256.563 & -43.035 & 63 & 561 & 10.8 & LMXB & $\mathrm{Y}$ & \\
\hline $4 \mathrm{U} 1705-440$ & 257.226 & -44.102 & 78 & 805 & 14.5 & LMXB & $\mathrm{Y}$ & \\
\hline GX 354-0 & 262.989 & -33.834 & 61 & 1977 & 18.6 & LMXB & $\mathrm{Y}$ & \\
\hline GX $1+4$ & 263.009 & -24.745 & 64 & 805 & 7.8 & LMXB & $\mathrm{Y}$ & \\
\hline $4 \mathrm{U} 1735-444$ & 264.742 & -44.450 & 47 & 272 & 11.8 & LMXB & $\mathrm{N}$ & \\
\hline 1E1740.7-2942 & 265.978 & -29.745 & 47 & 1251 & 11.2 & LMXB & $\mathrm{Y}$ & \\
\hline IGR J17464-3213 & 266.565 & -32.233 & 134 & 536 & 23.1 & LMXB & $\mathrm{Y}$ & \\
\hline IGR J17473-2721 & 266.825 & -27.344 & 37 & 129 & 5.3 & LMXB & $\mathrm{N}$ & X-ray burst \\
\hline GX $3+1$ & 266.983 & -26.563 & 40 & 276 & 10.0 & LMXB & $\mathrm{Y}$ & \\
\hline AX J1749.1-2639 & 267.300 & -26.647 & 196 & 1097 & 37.2 & HMXB & $\mathrm{Y}$ & \\
\hline SAX J1750.8-2900 & 267.600 & -29.038 & 67 & 137 & 21.8 & LMXB & $\mathrm{N}$ & X-ray burst \\
\hline SWIFTJ1753.5-0127 & 268.368 & -1.453 & 116 & 158 & 6.0 & LMXB & $\mathrm{N}$ & \\
\hline GX 5-1 & 270.284 & -25.079 & 137 & 2621 & 33.4 & LMXB & $\mathrm{Y}$ & \\
\hline GRS 1758-258 & 270.301 & -25.743 & 100 & 791 & 28.2 & LMXB & $\mathrm{N}$ & \\
\hline GX 9+1 & 270.385 & -20.529 & 50 & 1260 & 8.4 & LMXB & $\mathrm{N}$ & \\
\hline SAX J1808.4-3658 & 272.115 & -36.979 & - & - & - & LMXB & $\mathrm{N}$ & X-ray burst \\
\hline SGR 1806-20 & 272.164 & -20.411 & - & - & - & SGR & $\mathrm{N}$ & Short bursts \\
\hline SAX J1810.8-2609 & 272.685 & -26.150 & 35 & 376 & 8.4 & LMXB & Y & \\
\hline GX $13+1$ & 273.631 & -17.157 & 46 & 371 & 5.5 & LMXB & $\mathrm{Y}$ & \\
\hline $1 \mathrm{M} \mathrm{1812-121}$ & 273.800 & -12.083 & 45 & 708 & 7.8 & LMXB & $\mathrm{Y}$ & \\
\hline GX $17+2$ & 274.006 & -14.036 & 122 & 2705 & 22.4 & LMXB & Y & \\
\hline 4U 1820-303 & 275.919 & -30.361 & 80 & 3099 & 26.6 & LMXB & Y & \\
\hline 3 A $1822-371$ & 276.445 & -37.105 & 53 & 1016 & 7.9 & LMXB & $\mathrm{Y}$ & \\
\hline Ginga 1826-24 & 277.367 & -23.796 & 84 & 3316 & 17.3 & LMXB & $\mathrm{Y}$ & \\
\hline Ginga $1843+009$ & 281.412 & 0.891 & 95 & 125 & 5.2 & HMXB & $\mathrm{N}$ & \\
\hline HETE J1900.1-2455 & 285.036 & -24.920 & 60 & 210 & 7.6 & LMXB & Y & \\
\hline GRS $1915+105$ & 288.798 & 10.946 & 347 & 4361 & 66.8 & LMXB & $\mathrm{Y}$ & \\
\hline $4 U 1954+31$ & 298.926 & 32.097 & 64 & 279 & 13.8 & LMXB & $\mathrm{Y}$ & \\
\hline Cyg X-1 & 299.590 & 35.202 & 714 & 7923 & 161.4 & HMXB & $\mathrm{Y}$ & \\
\hline EXO $2030+375$ & 308.063 & 37.638 & 92 & 1976 & 28.7 & HMXB & $\mathrm{Y}$ & \\
\hline Cyg X-3 & 308.107 & 40.958 & 165 & 6290 & 32.8 & HMXB & $\mathrm{Y}$ & \\
\hline Cyg X-2 & 326.172 & 38.322 & 59 & 3793 & 16.1 & LMXB & $\mathrm{Y}$ & \\
\hline
\end{tabular}

Notes. Average flux (mCrab, 20-60 keV), total exposure and maximum detection significance are based on daily averages. A "Y" in the "orbital data" column specifies that the source was also detected on an orbital timescale, while the Note column identifies specific integrations (e.g., short transients). The source type is identified according to the following classes: HMXB - High mass X-ray binary; LMXB - Low mass X-ray binary; SGR - soft gamma repeater; PWN - pulsar wind nebula; Sy - Seyfert galaxy; BL - BL Lac; RG - radio galaxy; AXP - Anomalous X-ray pulsar.

typical results of the scientific analysis of the SuperAGILE data in two different conditions.

In Fig. 5, we provide the set of two one-dimensional sky images obtained by a one-day integration centered on RA $=149.31$,
Dec $=-62.95(\mathrm{~J} 2000$, corresponding to Galactic coordinates $l=284.55, b=-6.48)$ on 10 January 2008 . The abscissae report the angular offset with respect to the center of the SA field of view, in the $X$ and $Z$ coordinates of the SA reference frame 


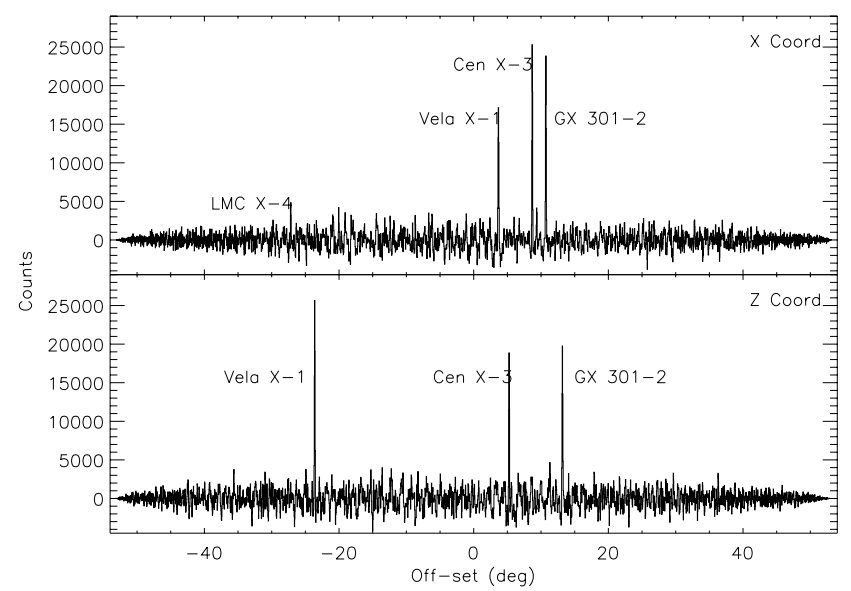

Fig. 5. One-day exposure of the Vela field. Data refer to MJD 54475 (10 January 2008). Center of the field of view $(X, Z)=(0,0)$ is at $\mathrm{RA}=149.31$, Dec $=-62.95(\mathrm{~J} 2000)$, corresponding to Galactic coordinates $l=284.55, b=-6.48$.

( $+Z$ is the Sun direction, $+Y$ is the pointing direction). The bin size is the SA pixel, corresponding to 3 arcmin at the center of the field of view. The ordinates show the counts per bin for the sum of the two detectors encoding the same direction $(X$ or $Z)$, after a deconvolution with the mask code. With reference to the "two-dimensional encoding" caused by the spacecraft attitude variations (see Sect. 2), the most appropriate representation of a SA sky image, for each of the $X$ and $Z$ coordinates, would be two-dimensional, similar to Fig. 2, where the proper attitude correction is applied to every region in the field of view. However, for the sake of clarity we chose to present one-dimensional images, displaying the on-axis non-coding zone for every sky bin (i.e., $X=0$ for the $\mathrm{Z}$ image, and viceversa), except for those where a source is detected, for which the relevant non-coding zone correction is shown, to image the detected source properly. Thus, these one-dimensional images of the sky are a sort of mosaic of different non-coding zones.

The ordinates of these images report the integrated counts for each bin. According to the shape of the SA PSF, in most cases the source peak in the counts image corresponds to a fraction varying between $70 \%$ and $100 \%$ of the total source counts (see Evangelista et al. 2006, for details). The estimation of the total source counts then needs to be completed by proper integration over the PSF shape at the position of the source.

It is useful to note how the amplitude of the fluctuations in Fig. 5 decreases as the off-axis angle increases. This is because the coded imaging subtracts the background counts but not their fluctuations, which depend on the total counts (background plus sources) in the fraction of the detector exposed at each specific off-axis angle. In other words, the on-axis direction is seen by the full area of the detectors, while increasingly off-axis directions are observable by decreasing fractions of it. This implies that there are a smaller number of counts in the involved detector region, and then lower values of their fluctuations. Consequently, to infer the statistical significance of any peak in the image, the counts in the peak must be compared to the value of the background fluctuations (e.g., $\sigma$ ) at that specific position in the field of view.

The one-day integration of the Vela field taken on MJD 54475 detected of a few sources in both X and Z SA detectors, noticeably Vela X-1, Cen X-3, and GX 301-2, with an estimated average flux of about 380,130 , and $195 \mathrm{mCrab}$, respectively. The detection significance was 14,22 and $21 \sigma$ in the

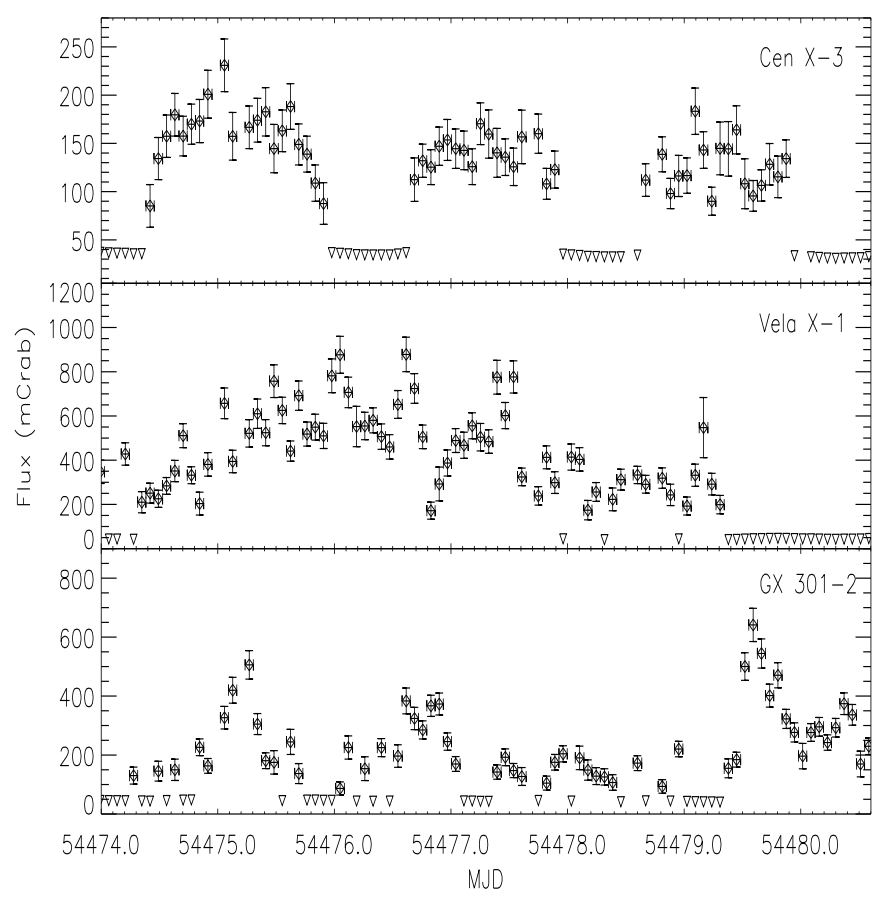

Fig. 6. SuperAGILE simultaneous orbital light curves for Vela X-1, Cen X-3 and GX 301-2 between MJD 54474 and MJD 54480. Each time bin corresponds to one AGILE orbit (approximately 100 min real time). Upside-down triangles identify 3- $\sigma$ upper limits when there was no detection. Vertical error bars are $1-\sigma$ uncertainties, and include systematics.

$X$ coordinate image, and 27,17 , and $19 \sigma$ in the $Z$ coordinate, respectively. The net exposure to each of the sources in this image is 46,56 and $59 \mathrm{ks}$, respectively. We note the wide angular separation between the sources, of up to $\sim 40^{\circ}$.

When looking at the SA images of the sky, it is important to rcall how the angular response of the experiment works (see Feroci et al. 2007, for details). The SA FOV is defined by a collimator that causes a linear decrease in the effective area from the center of the field to the edge. The shape of the FOV is rectangular (see Fig. 1) and is narrower in the non-coding direction, implying that the effective area decreases more rapidly if a source "moves" from the center to the edge of the FOV along the noncoding direction than along the coding one. Since the peaks in the SA images are in counts, the shape of the collimator response ensures that the peak value for a given source is strongly dependent on its position in the FOV. This effect explains why the same two sources, observed simultaneously, may exhibit peaks of very different relative heights in the $\mathrm{X}$ and $\mathrm{Z}$ images, being dependent on their relative positions in the $X$ and $Z$ SuperAGILE coordinates. An example of this effect is given by Cen $X-3$ and Vela X-1 in Fig. 5: the peak of Vela X-1 is higher in the Z image than in the $X$, because in the latter it lies at a position of smaller effective area. The inverse situation applies to Cen X-3. This SA property also clarifies why the source LMC X-4 is detected significantly only in the $\mathrm{X}$ image. The source is expected to be at a position in the SA reference frame of $(X, Z)=(-27.14,-0.87)$ degrees. At this position, the exposed area of the $\mathrm{Z}$ detectors (for which the non-coding position is $\sim 27^{\circ}$ off-axis) is insufficient for source detection, while in the $\mathrm{X}$ detectors it is detected at $10 \sigma$, with a flux of $70 \mathrm{mCrab}$.

Given the high detection significance of the sources in the daily image integration, light curves can be obtained with finer time resolution. In Fig. 6, we show the light curve of the same 

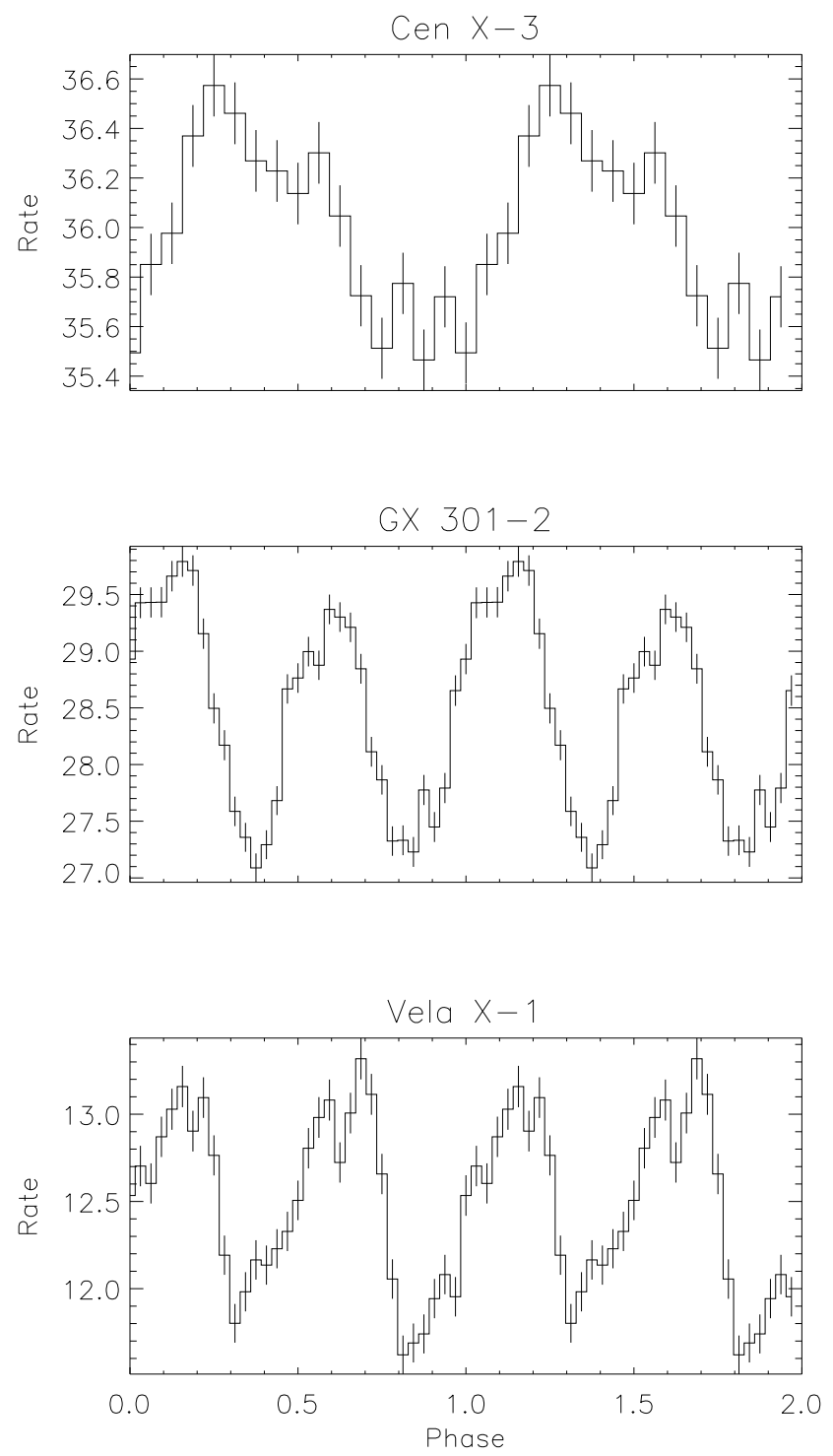

Fig. 7. SuperAGILE folded light curves for the X-ray pulsars Vela X-1, Cen X-3, and GX 301-2. Data used here are from MJD 54753 for Vela X-1, 54317 for Cen X-3 and GX 301-2.

sources, obtained by the automated orbital analysis, over a time span including MJD 54475, with a bin size corresponding to the SA orbital timescale ( $\sim 6 \mathrm{ks}$ elapsed time). The plot shows the average flux measurement obtained by SuperAGILE at each AGILE orbit, with a net exposure for each of the sources of about $\sim 3-4000 \mathrm{~s}$. In contrast to the images, we note that in the light curves the source counts are normalized and directly represent flux values (based on the assumption of a Crab-like energy spectrum). When the source is not detected, the 3- $\sigma$ upper limit at the position of the source is provided. It is interesting to note, for example, how the $\sim$ half-day binary eclipse is clearly detected in the light curve of Cen X-3, every $\sim 2.1$ days.

Because the photon-by-photon data are always transmitted to the ground (see Sect. 2), a more detailed analysis of individual sources can be carried out provided that the data are of high enough statistical quality. As an example, for the three X-ray binary pulsars in the Vela field for which we provided images and light curves above, SuperAGILE was able to detect their spin periodicity and obtain their pulse profile by period folding. In Fig. 7, we show the folded light curves at the periods $P \simeq 283 \mathrm{~s}$ for Vela X-1, $P \simeq 680 \mathrm{~s}$ for $\mathrm{GX} 301-2$ and $P \simeq 4.8 \mathrm{~s}$ for Cen $\mathrm{X}-3$ (the folding epoch is arbitrary).

\subsection{The Galactic center}

The Galactic center region is the field most rich in sources for any X-ray experiment. A number of bright sources are concentrated within the central $\sim 10-20^{\circ}$ of our Galaxy, mostly neutron star and black-hole X-ray binaries, and every search of this region offers a different picture, because of their significant variability. For SuperAGILE, this field is also very complex, because of the one-dimensional encoding of the sky, leading to source confusion in some conditions. This is even more difficult because of the large field of view of SA, which often includes Sco X-1, the brightest X-ray source in the sky, despite its $\sim 23^{\circ}$ Galactic latitude. Images taken by SA of this region of the Galaxy need to be analyzed very carefully, especially when Sco X-1 is in the field of view because of the higher fluctuations and coding noise it produces.

The Galactic center region was observed 5 times in 2007-2008 (see Table 1): two short observations in 2007 (August and October), and two one-month pointings in 2008 (March and September) and March 2009. In Fig. 8, we show a SuperAGILE image taken on October 2007 of a field centered close to the Galactic center (boresight at $l=21.430$, $b=-20.031)$ such that the position of Sco X-1 is at SA coordinates $X=-6.252, Z=48.792$, which is outside the field of view of the $X$ detectors (for these detectors it has a noncoding offset angle of $\sim 49^{\circ}$, and their effective area goes to zero at $\sim 35^{\circ}$ offset) and marginal in $\mathrm{Z}$. The good condition of having no bright sources in the field allowed us to complete a 9-day long integration, corresponding to a typical net exposure of the sources of about $370 \mathrm{ks}$. The X image (Fig. 8, top panel) is nearly parallel to the Galactic longitude (i.e., the Galactic plane is seen "face-on") and it contains several sources, as labelled in the figure: GRS 1915+105, 1M 1812-121, GX 17+2, GX 13+1, Ginga 1826-24, GX 5-1, SAX J1810.8-2609, 4U 1820-303, 3A 1822-371, and AX J1846.4-0258 and/or XTE J1855-026 (confused, being projected onto $X$ coordinates separated by less than 0.4 arcmin). These sources are all X-ray binaries in our Galaxy, and their intensity in this observation ranges from $\sim 40$ to $\sim 150 \mathrm{mCrab}$.

The sources in the $\mathrm{X}$ image are approximately evenly distributed over the field of view, thus the balance of counts does not cause significant (concentrated) coding noise disturbance. The Galactic plane projection onto the SA $Z$ coordinate is instead almost "edge-on" and the sources are clustered around an offset of $\sim 20-30$ degrees (the Galactic center is at $Z \simeq 28^{\circ}$ ). This and the bright Sco X-1 being about $49^{\circ}$ off-axis, induces significant fluctuations related to coding noise at the opposite side of the field of view, near an offset of $-20^{\circ}$. The sources that we identified in the $\mathrm{Z}$ image are: Ginga 1826-24, 4U 1820-303, 3A 1822-371, GX 17+2, GX 13+1, SAX J1810.8-2609, GX 5-1, GX 354-0, GX 1+4, and Sco X-1. These sources are not all identical to those detected in the $\mathrm{X}$ image, because of their different projections on the two SA coordinates. As an example, the microquasar GRS $1915+105$ lies at SA coordinates $X=-30.607$, $Z=2.594$, implying that it has a reasonably exposed area in the $X$ detectors (being only $2.6^{\circ}$ off-axis in their non-coding direction) and a negligible area with the $\mathrm{Z}$ detectors (for which it is at $30.6^{\circ}$ in the non-coding direction). Thus, in the $\mathrm{Z}$ image we expect a far less significant peak for this source, which is indeed detected in the expected position but with a significance below our threshold. Similarly, the two sources confused in the 


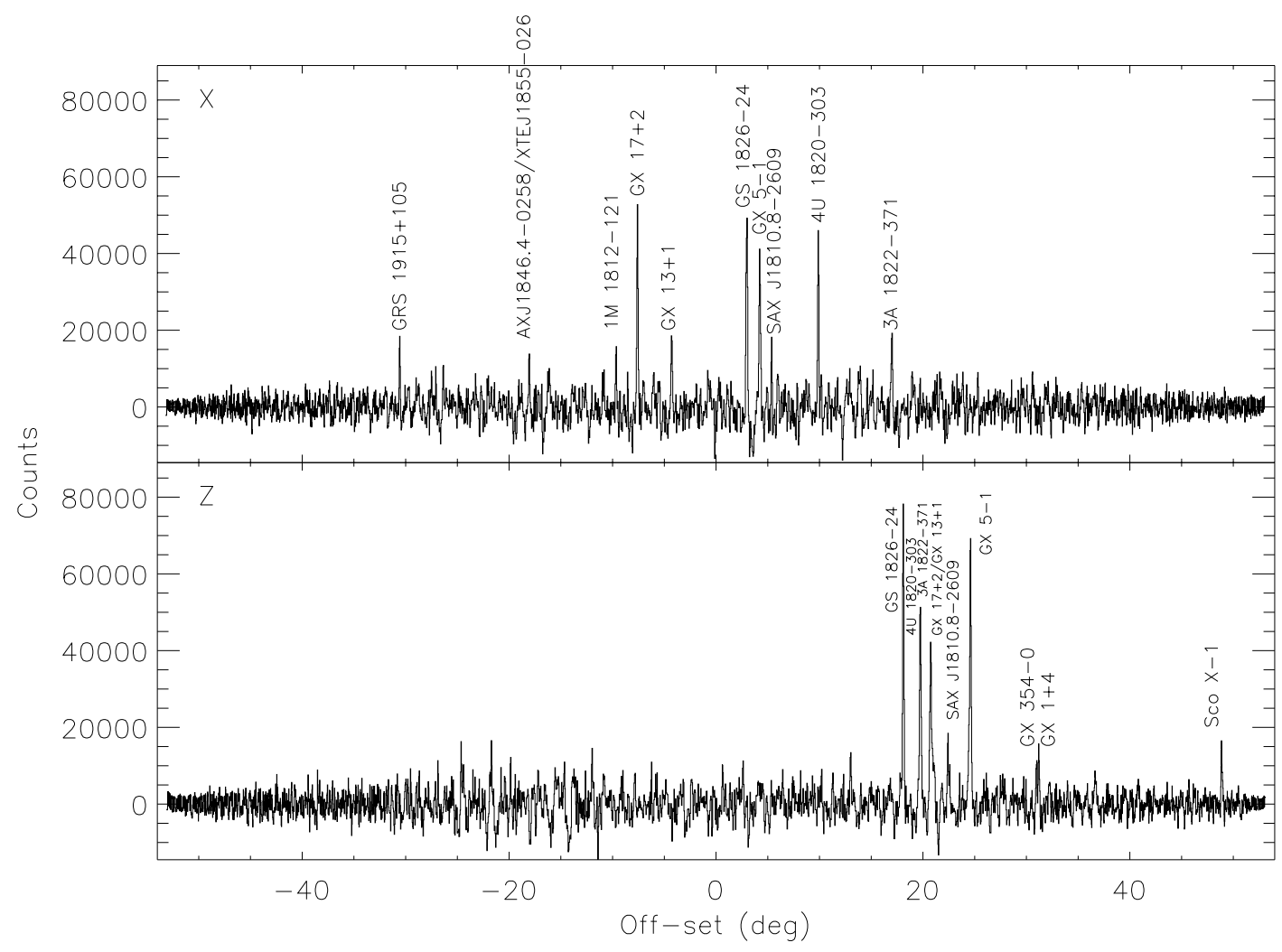

Fig. 8. A $600 \mathrm{ks}$ long exposure to a field near to the Galactic Center, from 13th to 22nd October 2007. The field is centered at RA $=296.265$, $\mathrm{Dec}=-18.840(l=21.430, b=-20.031)$. In these images, the Galactic Center $(l=0, b=0)$ is at SuperAGILE coordinates $X=8.7, Z=28.1$.

$\mathrm{X}$ image, at $X=-18.03$, AX J1846.4-0258 and XTE J1855026 , are expected at $Z=12.293$ and $Z=9.873$, respectively. Although their coordinates are not consistent with a detection in the $Z$ image, the presence of a small peak at $Z=12.3$ and nothing at $Z=9.9$ supports the identification with AX J1846.40258. Additional weaker sources are probably detected in these images, but they will be detected significantly only after the coding noise because of the brighter sources will be subtracted by the future version of the SA data analysis software.

\subsection{Galactic transients}

In addition to the long-term monitoring of variable sources, the wide field of view and sensitivity of SuperAGILE allow the detection and localization of short timescale events, originating in Galactic transient sources. This is the case for: the short bursts from soft gamma repeaters (SGRs), which have typical durations of a few hundreds of ms; the type I X-ray bursts from low mass $\mathrm{X}$-ray binaries (LMXB), in the range of tens of seconds, and the outbursts or flares from high mass X-ray binaries (HMXB), usually lasting from hours to days.

The relatively small fraction of time spent by AGILE pointing at the Galactic center region (see Sect. 4 and Table 1), where these transients are more frequent, meant that the SA detections were rather infrequent. Type I X-ray bursts, which have thermal energy spectra with temperatures of about $k T \sim 2-$ $3 \mathrm{keV}$, are also difficult to the detect because of the bandpass of SA. Type I X-ray bursts were detected by SA from IGR J17473-2721, SAX J1750.8-2900, and the millisecond pulsar SAX J1808.4-3658 (Del Monte et al. 2008b; Pacciani et al. 2008b; Del Monte et al. 2008c). The case of IGR J17473-2721 is noticeable because the single X-ray burst detected by SA was the first one ever detected from this previously unidentified source and allowed its classification as a LMXB. This was confirmed by the later activity of the source detected by other experiments: the complete outburst lasted for approximately 3 months, exhibiting several bursts and an hard-to-soft spectral state change before reverting back to a hard spectral state. During the soft spectral state, some $900 \mathrm{~Hz}$ QPOs were also detected with the RossiXTE/PCA, as is typical of atoll sources (Altamirano et al. 2008).

Longer outbursts were detected from several X-ray binary systems: the Compton-thick X-ray binary source IGR J163184848 (Pacciani et al. 2008c), the Be binary GRO J1008-57 (Evangelista et al. 2008), the HMXB 1A 1118-61, the slowly spinning HMXB source 3A 0114+650 (Pacciani et al. 2009b), and the neutron star transient SAX J1750.8-2900 (Pacciani et al. 2008d, see Fig. 9). The recurrent transient and black hole candidate IGR J17464-3213 was detected by SA on September 2008. An interesting state of Vela X-1 was also detected by SA on October 2008 (Soffitta et al. 2008): the source flux rose from an average $500 \mathrm{mCrab}$ level up to more than 2 Crab in approximately $7 \mathrm{~h}$, before redescending to a $500 \mathrm{mCrab}$ flux. The intensity of the source during the flare was such that the individual 283.5-s pulsations of the neutron star were clearly visible on the raw 16-s SA ratemeters counters. Interestingly, during the hourlong flare, shorter $\sim 100 \mathrm{~s}$ flares were also observed, peaking at fluxes as high as 4 Crabs.

During the past two years, several magnetar sources entered new periods of intense bursting activity, namely the SGRs 1806-20, 1627-41, and 0501+4516 (discovered by Swift/BAT on 22nd August 2008, Barthelmy et al. 2008), as well as the anomalous X-ray pulsar (AXP) 1E 1547.0-5408. Unfortunately, the periods of activity of these sources generally 


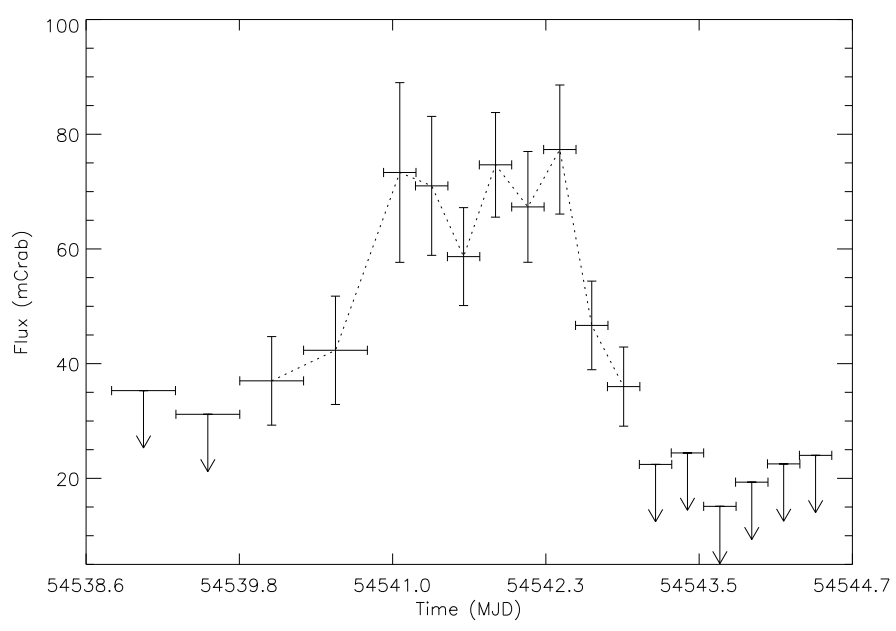

Fig. 9. A $\sim 1$ day flare from the neutron star transient SAX J1750.82900 detected by SuperAGILE on March 16-17, 2008 (Pacciani et al. 2008d). Arrows show $3 \sigma$ upper limits, when no significant detection is available.

did not coincide with the observation of their sky regions by AGILE. Thus, only a handful of short bursts were imaged by SA, originating in SGR 1806-20, 1E 1547.0-5408, and SGR 0501+4516. The latter was indeed monitored during a dedicated target of opportunity observation approximately 10 days after its discovery, from 31st August to 10th September, but the source turned out to have gone back to an almost burst-quiescent state (see, e.g., Rea et al. 2009) and only 2 short bursts were detected by SuperAGILE (Feroci et al. 2008b). Examples of short bursts from the SGR sources detected by SA are shown in Fig. 10, highlighting the time resolution achieved by the photon by photon data, basically limited by the counts statistics only. Sometimes the short bursts from these active magnetars were so bright that they were detected in the SuperAGILE rates even when the source was located well outside the experimental field of view, after passing through the collimator walls or the AGILE satellite structures. This happened for a few events from SGR 1806-20, SGR 0501+4516, and 1E1547.0-5408.

\subsection{Gamma ray bursts}

Gamma ray bursts (GRBs) are among the primary scientific targets of AGILE, which is the first gamma-ray mission since EGRET, in the "afterglow era" of GRB science. Based on what is known from EGRET about this field, GRB emission in the energy range above $50 \mathrm{MeV}$ is relatively uncommon for ordinary GRBs (involving the few percent of the brightest bursts), and when present, the expected number of photons is such that an accurate localization in the gamma-ray range only is difficult with an experiment of $\sim 500 \mathrm{~cm}^{2}$ effective area such as the AGILE/GRID, although for very bright events the shorter deadtime of GRID compared to EGRET enables the collection of a higher number of photons. To discover the multiwavelength GRB afterglow (e.g., soft X-rays, optical, infrared, and radio), and the source distance thereof, a prompt arcmin-level localization of the GRID-detected GRBs is highly desirable. SuperAGILE is in the strongest position in this respect, its field of view covering a large fraction of the AGILE/GRID at any time.

SA carried out this mission quite diligently. About 1 GRB/month was discovered and localized by SA, with typical error radii of 3 arcmin (except for those 1-2 events localized at

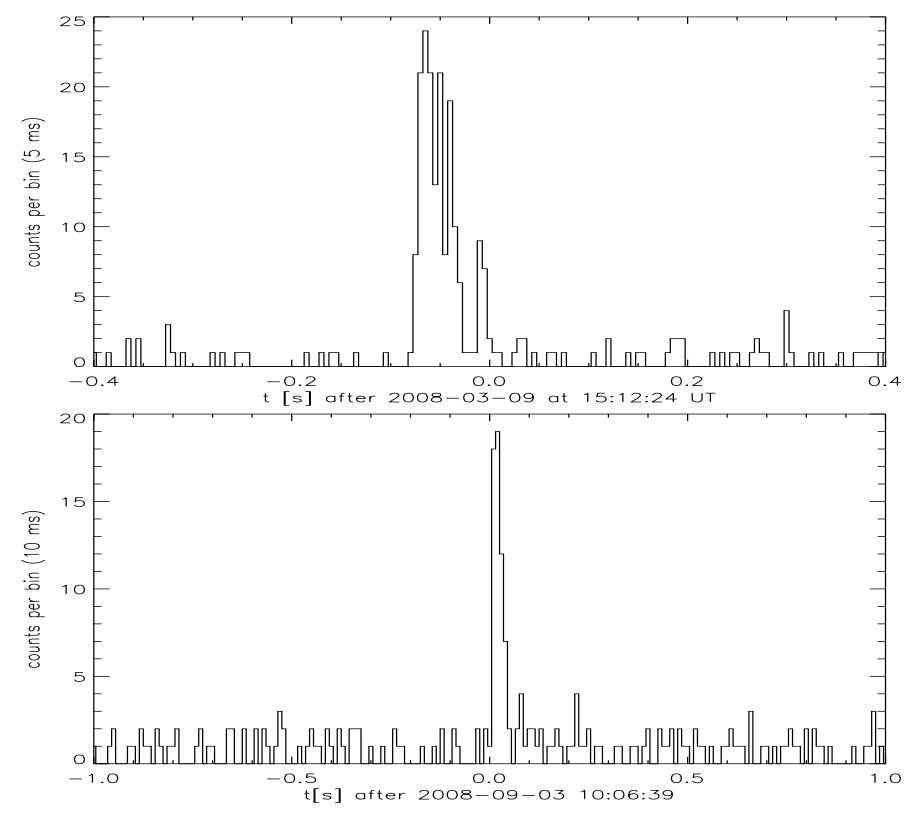

Fig. 10. Short bursts from SGR 1806-20 (top panel) and SGR $0501+4516$ detected and localized by SuperAGILE. The time resolution is limited by the number of detected events, but allows to detect $<5 \mathrm{~ms}$ structures in the burst from SGR 1806-20.

the very beginning of the mission). Most of them were followedup by the Swift X-ray telescope, which discovered their X-ray afterglow, thus also indirectly confirming the accuracy of the SA GRB positions. The first GRB localized by SA was detected on 24 July 2007, during the AGILE science verification phase. An extensive report on this event may be found in Del Monte et al. (2008a), while a description of the SA GRB triggering system may be found in Del Monte et al. (2007).

When a bright GRB illuminates the SA detectors, during its short duration it usually overwhelms the background count rate. In Fig. 11 (top panel), we show the case of one GRB, 080723B, where the peak count rate of the GRB exceeds by more than 5 times the average background rate recorded by the SA experiment. Taking into account the source-dominated condition and that on such a short timescale both the temperature and the satellite attitude variations become negligible, images of the event can be accumulated over the energy range from 17 to $60 \mathrm{keV}$, deriving the projections of the source position in the SA reference frame. They are shown in Fig. 11 (bottom panel) for the same event. During the GRB emission, the burst intensity typically outshines any other source in the field. In a few cases where a bright field source has an intensity comparable to that of the GRB on the same timescale, that source is usually easily identified, because it is among the few brightest X-ray sources in the Galaxy (e.g., Sco X-1, or Cyg X-1, or others). From these images, an unambiguous sky position for the GRB is derived and promptly distributed to the world-wide community by means of the GCN coordinate distribution network ${ }^{3}$.

The case of GRB 080723B is representative of the most typical in terms of the SA GRB detection statistics. However, in some cases the event occurs in the part of the SA field of view with only one-dimensional imaging encoding. In this case, the SA imaging response may only be a narrow $( \pm 3$ arcmin) strip in the sky, whose length depends on the geometry of the SA field of view and Earth occultation constraints. Typically, it is of the order of $\sim 10^{\circ}$ or more. This can be reduced to

3 http://gcn.gsfc.nasa.gov/gcn3_archive.html 

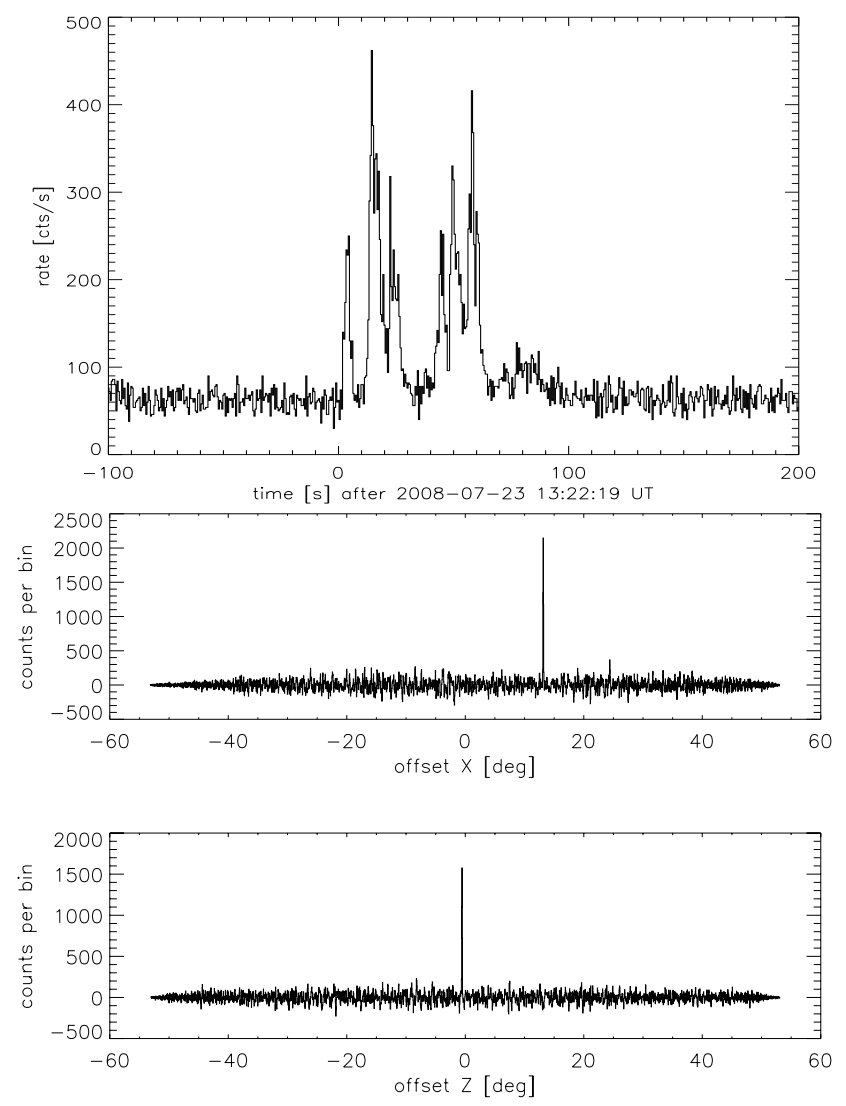

Fig. 11. Light curve (top panel) and images (bottom panel) of GRB 080723B, as observed by SuperAGILE. The bin size in the light curve is $0.5 \mathrm{~s}$.

arcmin size by including the analysis of the delay in the arrival time of the event at both the SA location and some other longdistance spacecraft (i.e., a few light-seconds), within the context of the Interplanetary Network (e.g., Hurley 2008). This was the case, for example, of GRB 080514B, which was localized by SA at $X=-37.61^{\circ}$, then in the $1 \mathrm{D}$ coding region, and the error box was reduced to a $100 \operatorname{arcmin}^{2}$ sky region by means of a rapid triangulation with the data of the GRB detector onboard Mars Odyssey. This event was especially noticeable because it was also the first GRB detected above $25 \mathrm{MeV}$ by the AGILE GRID. It was indeed the first GRB detected at high energies after EGRET, and the first ever GRB with emission above $25 \mathrm{MeV}$ associated with a multiwavelength afterglow and a photometric redshift of $z=1.8$ (Giuliani et al. 2008a; Rossi et al. 2008).

\subsection{Extragalactic sources}

The bandpass and sensitivity of SA are certainly not optimal for the study of extragalactic sources. Only a few bright AGNs were detected during the reported period (see Table 2), noticeable cases being the radiogalaxy Cen $\mathrm{A}$, for which several detections were obtained also on the orbital timescale, and the BL Lac source Mkn 421. The latter was serendipitously observed during a one-week AGILE target-of-opportunity observation toward the blazar W Comae. Mkn 421 was detected in a hard X-ray flaring state with a flux peaking at approximately $55 \mathrm{mCrab}$ (Costa et al. 2008). The SA observation of the hard X-ray flaring motivated a target-of-opportunity observation with Swift/XRT that measured the source flux at its brightest state ever at soft X-rays. An integration over the entire week of data also resulted in a detection above $100 \mathrm{MeV}$ by the AGILE/GRID. The SA and GRID data were then analyzed in a multi-frequency context, from optical to $\mathrm{TeV}$ energies (Donnarumma et al. 2009a).

\subsection{Monitoring of AGILE GRID gamma-ray sources}

The classes of persistent sources detectable in gamma-rays with the sensitivity of the AGILE GRID (e.g., blazars, gamma-ray pulsars, supernova remnants, etc.) are usually distinct from those typically detectable in hard X-rays with the sensitivity of SA (e.g., low and high mass X-ray binaries), although there are exceptions, e.g. the reported observations of Mkn 421 or the Crab pulsar (Pellizzoni et al. 2009), or the AGILE observation of the flat spectrum radio quasar 3C 373 (Pacciani et al. 2009a). However, the SA data are searched systematically for hard X-ray excesses in time and space coincidence with those detected by the AGILE/GRID (and vice-versa). This has measured so far mostly upper limits. In some cases, the SA upper limits provide significant constraints on the interpretation of the gamma-ray data. As an example, the rapid and bright gamma-ray transients detected by the AGILE GRID in the Galactic plane (e.g., Longo et al. 2008; Pittori et al. 2008; Chen et al. 2008; Giuliani et al. 2008 b) are unknown in origin. They were typically observed on the timescale of one day, and no contemporaneous observations are available in other energy ranges. SuperAGILE observed those sources simultaneously with the gamma-ray detections but did not find any coincident X-ray emission. The value of the upper limits depends on the source position in the SA field of view and the integration time, and ranges between 10 and $45 \mathrm{mCrab}$. The gamma-ray sources, instead, displayed emission as high as 0.5 Crab above $100 \mathrm{MeV}$.

\section{Public distribution of SuperAGILE light curves}

The orbital light curves of the sources detected by SA, which are automatically extracted by the SASOA pipeline, provide new input to the SA source database, after the processing of the latest available data following every passage of the AGILE satellite over the Malindi ground station. The orbit-averaged source fluxes in the standard 20-60 keV energy range are then calculated and automatically made publicly available to the general community, with no restrictions. The source flux data can be accessed from the SuperAGILE web page at AGILE Data Center ${ }^{4}$ for display and download purposes. Since they are the products of the automatic orbital source extraction, as we discussed in Sect. 2 the detection quality has not been optimized, but it is usually good enough for the monitoring of the intensity state of bright sources. The ASDC web page is automatically updated twice a day, and the availability of the new data for public distribution is then not delayed by any more than $12 \mathrm{~h}$.

Orbital flux measurements are provided for only a subset of the sources listed in Table 2, those for which significant detections could be obtained by the orbital analysis (identified with "Y" in the "orbital data" column of the table). The brightest sources are frequently detected, while weaker sources (or sources that have only been marginally observed) have far poorer coverage. Any source can be monitored only when it is inside the SuperAGILE field of view. Thus, a source light curve available from the web usually consists of clustered sets of measurements obtained by subsequent orbits in the observing periods, interleaved with long gaps corresponding to periods when the AGILE satellite was pointing at other regions. As an example,

${ }^{4}$ http://agile.asdc.asi.it/sagilecat_sources.html 

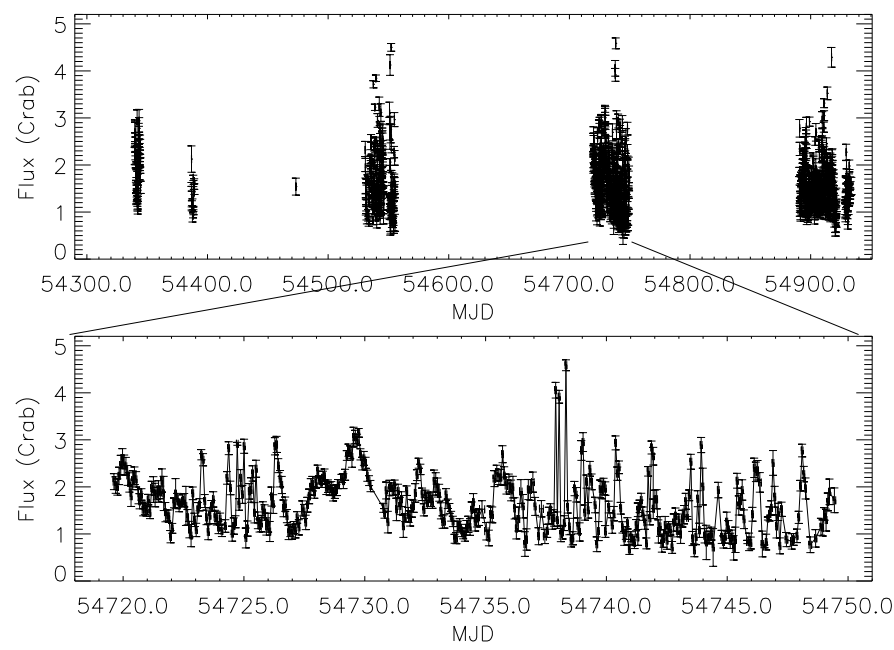

Fig. 12. Long-term orbital light curve of the very bright X-ray binary Sco X-1 as it is available from the SA web page at the ASDC web site. Top panel shows the complete data set, from July 2007 to April 2009. Bottom panel is a zoom of a one-month time interval, showing the short term ( hours) variability of the source.

in the top panel of Fig. 12 we show the complete orbital data set for the bright source Sco X-1 (as available from the public website as of April 2009), while the bottom panel shows the same data zoomed over a one-month period (September 2008). From the same website, the user can access the AGILE pointing plan to discover when the source of interest was or will be in the SuperAGILE field of view.

The web page reports only the positive detections (i.e., of significance greater than $4 \sigma$ in either of the $X$ or $Z$ detectors). This means that if the source flux decreases below the detection limit for one orbit, this will correspond to a missing point in the light curve. This may also sometimes be caused by a very short net exposure time during a given orbit, e.g., when the source visibility (with respect to the Earth occultation) overlaps with the satellite passage through the South Atlantic Anomaly. These conditions can usually be recovered by a human-assisted analysis, optimizing the source exposure. It is worth emphasizing again here that being based on positive detections only, the SA light curves currently publicly available offer an intrinsically upward-biased view of the flux history of the sources, except for very bright sources (i.e., above $\sim 100-200 \mathrm{mCrab}$ ).

Non-positive detections and upper limits can also be computed, typically ranging between 10 and few tens of mCrab, depending on the integration time and the source position in the field of view. They are currently not yet provided at the web page due to computing time constraints, but can be provided for specific sources by the SA hardware team on request.

The automated orbital data processing applies filters to the source detections, mostly based on the exposed area of the source and the comparison of the source flux with a pre-set range, to guarantee the reliability of the real-time, automated, source-flux extraction. When a source detection is filtered out, it is automatically sent as an input to the manual processing, which verifies the detection quality and reliability. In the case of validation, the data are injected back to the public archive as soon as the human-validated analysis is available. In principle, this implies that the SA source flux data are always subject to improvements and refinements at short notice, although this condition is rather infrequent. The temporary filter is also used as an alert to the SuperAGILE team about special conditions concerning the detected sources, in some cases leading to rapid communication through, e.g., Astronomer's Telegrams ${ }^{5}$.

We recall that since the AGILE boresight drifts by $\sim 1^{\circ}$ per day, the same source will be seen by SA at different positions of the field of view (that is, potentially with a largely different effective area) even during the same pointing, of typical durations of a few weeks. A given source may then pass within 2-3 weeks of the same pointing from being detectable to undetectable, or viceversa, not only because of its flux but also its changing position within the experimental field of view.

The SA data that is accessible through the web page are currently only the results of the automatic orbital processing, described in Sect. 2. Data products derived from analyses of data on different timescales, either shorter for bursts or outbursts, or longer for the weaker sources, are currently not provided. A refined source analysis is also currently not provided. Work is in progress to provide in the near future additional and more refined products, including daily integrations.

\section{Summary and conclusions}

The SuperAGILE experiment has been operating successfully onboard the AGILE mission since 23 April 2007. In this paper, we have provided a description of the SA data and an overview of the main scientific results achieved in the first $\sim 20$ months of scientific observations. The main goals of SuperAGILE are the simultaneous hard X-ray observation of the central region of the field of view of the AGILE/GRID experiment, the prompt discovery and localization of gamma-ray bursts and Galactic transients, and the long-term monitoring of the brightest Galactic sources.

The aim of simultaneous hard X-ray and gamma-ray observations of the same field is to discover correlated variability of sources in these two energy ranges, and use the finer angular resolution of SuperAGILE to localize gamma-ray sources detected by GRID. This is the first time that an X-ray and a gamma-ray imager have systematically observed the same field simultaneously. Regrettably, this has not yet discovered any previously unknown correlated behavior of sources. The sources that SuperAGILE and GRID have detected simultaneously were already known to emit in both energy ranges, e.g., the Crab pulsar, or the AGNs 3C 273 and Mkn 421. However, the guaranteed simultaneous GRID and SuperAGILE observations represent the basic seed of multifrequency campaigns, which are often complemented with radio to $\mathrm{TeV}$ observations. This has led, for example, to the detection of a flaring state of the BL Lac source Mkn 421 and to the interpretation of the time variability of the simultaneous optical-to-TeV spectral energy distribution in terms of a rapid acceleration episode of the leptons in the jet (Donnarumma et al. 2009).

The primary goal is of course to search for positive detections in both instruments, but there are cases where upper limits in one or the other are also important to interpret the origin of the detected emission. This was the case for the unidentified transient sources discovered by AGILE/GRID in the Galactic plane, within the Cygnus and Musca regions (e.g., Longo et al. 2008; Pittori et al. 2008; Chen et al. 2008; Giuliani et al. 2008b). Following Romero \& Vila (2009), the SuperAGILE upper limits to the simultaneous hard X-ray emission of these transient gamma-ray sources imply a very high ratio of the gammaray to X-ray luminosity, placing strong constraints on their

\footnotetext{
5 http://www . astronomerstelegram.org
} 
interpretation in terms of emission from the jets of galactic microquasars, and in particular a dominant hadronic component in the jet.

Interesting results have also been obtained in the field of gamma-ray bursts. Despite the prompt SuperAGILE arcminlocalization of several GRBs, which have led to searches in time and spatial coincidence, in the reported period only two events were detected significantly by AGILE/GRID (Giuliani et al. 2008a, Moretti et al. 2009), including GRB080514B, the first GRB ever detected for which the gamma-ray emission can be correlated with an afterglow counterpart at a measured distance, and GRB 090401B. The detection of these events by all the AGILE instruments - SA (18-60 keV), GRID (>25 MeV), MCAL (350-700 keV) and ACS (>8 keV) - has also allowed us to measure a few-second delay of the emission above $25 \mathrm{MeV}$ with respect to that at lower energies. The observations of the Compton Gamma Ray Observatory, particularly for the EGRET and BATSE experiments, have enabled us to determine that the GRBs with a gamma-ray counterpart detectable above the EGRET sensitivity (that is similar to that of AGILE/GRID) belong to the brightest $5 \%$ of the BATSE GRB distribution, although the statistics are only for 5 events. Although the SuperAGILE GRBs are selected towards the bright end of the BATSE distribution, because of its smaller area, the 2 GRID detections of 21 GRBs localized by SuperAGILE by the end of April 2009 are still consistent with the approximate EGRET statistics.

The pointing strategy of the AGILE mission, typically consisting of long exposures, offers as a by-product a long-term monitoring of the same field by SuperAGILE, and as a drawback a very inhomogeneous sky coverage (see Fig. 4), in particular for the Cygnus and Vela sky regions. Although the analysis of the SuperAGILE data collected so far has not yet completed (see Sects. 2 and 5.1), about 60 sources were detected over the time period reported in this paper. As can be seen from Table 2, the vast majority of them are X-ray binaries, mostly low mass $\mathrm{X}$-ray binaries (LMXB).

Despite the inhomogeneity and incompleteness of the SuperAGILE exposure and data analysis, it may be useful to place the list of sources detected by SA in the context of the current scenario for hard X-rays. Currently, INTEGRAL/ISGRI and Swift/BAT offer the most complete sky surveys in the hard $\mathrm{X}$-rays. The list of sources detected by SuperAGILE given in Table 2 was obtained mainly by daily integrations. The sensitivity of SuperAGILE on this timescale is in the range of $\sim 15$ 20 mCrab. Applying a flux cut at $15 \mathrm{mCrab}$ to the ISGRI 3.5-year catalogue (20-40 keV, Bird et al. 2007) and to the BAT 22-month catalog (14-150 keV, Tueller et al. 2009), we found about 50 and 40 sources, respectively. Of these, in ISGRI, 38\% are HMXB and $57 \%$ are LMXB, while, in BAT, we find $31 \%$ and $59 \%$, respectively. From the list in Table 2, SA detected 57 persistent sources, of which $33 \%$ are HMXB and 58\% are LMXB. As expected, a large number of sources are found to be in common, being the bright tail of the $\log N-\log S$ distribution of persistent hard X-ray sources. Although the agreement is remarkably good, the above comparison should not be taken rigorously, because of a large number of important caveats about pointing strategy, type of the analysis, exposure, energy range, and intrinsic source variability. However, it is robust confirmation that the source detection of SuperAGILE is consistent with the sensitivity limit of the current analysis.

Taking the comparison (as well as the caveats) further, we applied the same type of selection to the source catalog of the BeppoSAX/Wide Field Cameras (WFC, Verrecchia et al. 2007).
This experiment operated in the $2-26 \mathrm{keV}$ energy range. Selecting the sources with a reported average flux of above $15 \mathrm{mCrab}$ in $2-10 \mathrm{keV}$, we found $\sim 80$ sources, of which $20 \%$ are HMXB and $73 \%$ are LMXB. A comparison between the type and number of X-ray binary sources brighter than $15 \mathrm{mCrab}$ below (in the WFC) and above (in SA, BAT and ISGRI) $20 \mathrm{keV}$ shows that approximately the same number of objects is found among the HMXB, while the LMXB decrease to one half above $20 \mathrm{keV}$. This suggests that the LMXB, as a class, tend to have energy spectra softer than the Crab (photon index $\sim 2.1$ ), while the HMXB tend, on average, to have harder spectra.

Many of the SA sources were detected on the orbital timescale, meaning that public light curves are available for them at the ASDC web page (see Sect. 6), which is updated daily with new data (when available). In this paper we have provided detailed explanations on how these light curves are extracted and based on which assumptions, thus offering an understanding of the limits of their scientific use. Other sources were detected in either longer integrations or short outbursts. For these sources no publicly accessible science products are currently yet available.

The main characteristics of the SA observation is its continuity and long duration. Some Galactic sources have been observed for probably the longest continuous stretches of time ever, allowing for monitoring the long-term variability of their hard $\mathrm{X}$-ray flux. The duration and continuity of the monitoring distinguishes the SA data from those of more sensitive experiments (e.g., Swift/BAT) that offer short and poorly sampled observations of individual sources, which sometimes miss short-term variability or events. In addition, the SA field is always observed simultaneously by the AGILE/GRID, thus offering the simultaneous measurement of the flux above $\sim 50-100 \mathrm{MeV}$.

The SuperAGILE experiment has been operating nominally since its switch-on in orbit in 2007, showing no signs of degradation or losses with time. The in-flight operation of the AGILE mission is expected to continue until at least mid-2011, subject to approval by the Italian Space Agency (ASI).

Acknowledgements. AGILE is a mission of the Italian Space Agency (ASI), with co-participation of INAF (Istituto Nazionale di Astrofisica) and INFN (Istituto Nazionale di Fisica Nucleare).

\section{References}

Altamirano, D., Galloway, D., Chenevez, J., et al. 2008, ATel, 1651 Barbiellini, G., Fedel, G., Liello, G., et al. 2002, Nucl. Instr. Meth. A, 490, 146 Barthelmy, S., Beardmore, A. P., Burrows, D. N., et al. 2008, GCN 8113 Bird, A. J., Malizia, A., Bazzano, A., et al. 2007, ApJS, 170, 175 Chen, A., Caraveo, P., Giuliani, A., et al. 2008, ATel, 1585 Costa, E., Del Monte, E., Donnarumma, I., et al. 2008, ATel, 1574 Del Monte, E., Costa, E., Di Persio, G., et al. 2007, Proceedings of the 5th SCINEGHE Workshop, Frascati Physics Ser., 45, 201, ed. A. Lionetto, \& A. Morselli [arXiv: 0712 . 1548]

Del Monte, E., Feroci, M., Pacciani, L., et al. 2008a, A\&A 478, L5 Del Monte, E., Evangelista, Y., Feroci, M., et al. 2008b, ATel, 1445 Del Monte, E., Evangelista, Y., Feroci, M., et al. 2008c, ATel, 1732 Donnarumma, I., Costa, E., Del Monte, E., et al. 2006, Proc. SPIE Conf., 6266, 36

Donnarumma, I., Vittorini, V., Vercellone, S., et al. 2009a, ApJ, 691, L13 Evangelista, Y., Costa, E., Del Monte, E., et al. 2006, Proc. SPIE Conf., 6266, 35

Evangelista, Y., Donnarumma, I., Del Monte, E., et al. 2008, ATel, 1619 Feroci, M., Costa, E., Soffitta, P., et al. 2007, Nucl. Instr. Meth. A, 581, 724 Feroci, M., Costa, E., Del Monte, E., et al. 2008a, Proc. SPIE Conf., 7011, 63 Feroci, M., Pacciani, L., Del Monte, E. , et al. 2008b, ATel, 1705 Giuliani, A., Mereghetti, S., Fornari, F., et al. 2008a, A\&A 491, L25 Giuliani, A., Caraveo, P., Chen, A., et al. 2008b, ATel, 1547

Hurley, K. 2008, Proc. 37th COSPAR Scientific Assembly, Held 13-20 July, in Montréal, Canada, 1299

Labanti, C., Marisaldi, M., Fuschino, F., et al. 2009, Nucl. Instr. Meth. A, 598, 470 


\section{Feroci et al.: Hard X-ray sky with SuperAGILE}

Lazzarotto, F., Costa, E., Del Monte, E., et al. 2008, Astronomical Data Analysis Software and Systems, in (UK, London: Kensington Town Hall), ed. W. Argyle Robert, S. Bunclark Peter, \& R. Lewis. James, ASP Conf. Ser., 394, Proceedings of the conference held 23-26 September, 593

Lazzarotto, F., Costa, E., Del Monte, E. et al. 2009, Astronomical Data Analysis Software and Systems, ed. D. Bohlender, P. Dowler, \& D. Durand, in Quebec City, Quebec, Canada. ASP Conf. Ser., Proceedings of the conference held 2-5 November, in press

Longo, F., Chen, A., Vercellone, S., et al. 2008, ATel, 1492

Moretti, E., Longo, F., Barbiellini, G., et al. 2009b, GRB Coordinate Network circular \#9069

Pacciani, L., Uberti, O., Del Monte, E., et al. 2008a, Nucl. Instr. Meth. A, 593, 367

Pacciani, L., Costa, E., Del Monte, E., et al. 2008b, ATel, 1775

Pacciani, L., Feroci, M., Del Monte, E., et al. 2008c, ATel, 1713

Pacciani, L., Costa, E., Del Monte, E., et al. 2008d, ATel, 1428

Pacciani, L., Donnarumma, I., Vittorini, V., et al. 2009a, A\&A 494, 49
Pacciani, L., Costa, E., Del Monte, E., et al. 2009b, ATel, 1917

Pellizzoni, A., Pilia, M., Possenti, A., et al. 2009, ApJ, 691, 1618

Perotti, F., Fiorini, M., Incorvaia, S., Mattaini, E., \& Sant'Ambrogio, E. Nucl. Instr. Meth. A, 556, 228

Pittori, C., Verrecchia, F., Santolamazza, P., et al. 2008, ATel, 1394

Pittori, C., Verrecchia, F., Chen, A. W., et al. 2009, A\&A, 506, 1563

Prest, M., Barbiellini, G., Bordignon, G., et al. 2003, Nucl. Instr. Meth. A, 501, 280

Rea, N., Israel, G. L., Turolla, R., et al. 2009, MNRAS, 396, 2419

Romero, G. E., \& Vila, G. S. 2009, A\&A, 494, L33

Rossi, A., de Ugarte Postigo, A., Ferrero, A., et al. 2008, A\&A, 491, L29

Soffitta, P., Costa, E., Del Monte, E., et al. 2008, ATel, 1782

Tavani, M., Barbiellini, G., Argan, A., et al. 2009, A\&A, 502, 995

Trifoglio, M., Bulgarelli, A., Gianotti, F., et al. 2008, Proc. SPIE Conf., 7011

Tueller, J., Baumgartner, W. H., Markwardt, C. B., et al. 2009, ApJS, in press [arXiv: 0903.3037v2]

Verrecchia, F., in 't Zand, J. J. M., Giommi, P., et al. 2007, A\&A, 472, 707 